\title{
ASYMPTOTIC ANALYSIS OF ACOUSTIC WAVES IN A POROUS MEDIUM: INITIAL LAYERS IN TIME*
}

\author{
JOSE DIAZ-ALBAN ${ }^{\dagger}$ AND NADER MASMOUDI ${ }^{\ddagger}$
}

To Dave Levermore on his 60th birthday with friendship and appreciation

\begin{abstract}
This is the first of a series of three papers which study acoustic waves governed by the linearized compressible Navier-Stokes equations in a porous medium. In particular, we want to analyze the simultaneous inviscid and high frequency limits of fluid flows in a porous medium. In this paper, we focus on the case of strongly viscous flow, namely fluid flow without the presence of boundary layers in space. We study the behavior of the energy using two-scale expansions in space and reveal that initial layers in time trap the energy carried by the flow during the usual two-scale homogenization process. We examine the time-space boundary layer problem in our forthcoming works.
\end{abstract}

Key words. Acoustic waves, compressible Navier-Stokes, porous medium, boundary layers.

AMS subject classifications. 35B27, 35B40, 76N20, 76M50.

\section{Introduction}

There are many works that study the homogenization of the Stokes operator and that of the incompressible Navier-Stokes equations in a porous medium (open set perforated with tiny holes). We refer the interested reader to text books $[3,18,11]$ for some formal developments and to $[19,1,14]$ for some rigorous mathematical results. However, there are fewer works dealing with the homogenization of the compressible Navier-Stokes system, see for instance [8, 13, 9, 15, 20].

In this paper, we extend some of the methods developed in the incompressible case to study the case of the linearized compressible Navier-Stokes system. This paper (as well as the companion papers $[7,6]$ ) can be considered as the linear version of [13]. In [13], the goal was just to pass to the weak limit in the system and recover the porous medium equation. There, the main difficulty was the passage to the limit in the non linear terms. Here, we consider the linearized problem with different possible viscosities. Our main goal here is to give a complete description of the energy carried by the flow (and not just the weak limit).

Physically, it is well known that viscous flow problems under a Dirichlet boundary condition exhibit a thin layer forming at the boundary when viscosity goes to zero, separating the flow into two regions: a boundary layer and interior flow. The changes that occur in the flow within the boundary layer are very important in understanding the overall behavior of the fluid. Moreover, the viscous effects in space influence the behavior of the flow near initial time, and describing the changes of the flow near initial time is essential in describing the energy carried by the flow. In all our papers, time and/or space boundary layer phenomena take place and it is crucial to provide appropriate asymptotic expansions describing the flow in all regions of time and space.

\footnotetext{
*Received: April 13, 2011; accepted (in revised version): July 14, 2011.

${ }^{\dagger}$ Courant Institute, New York University, 251 Mercer Street, New York, NY 10012, USA (josediaz@cims.nyu.edu).

${ }^{\ddagger}$ Courant Institute, New York University, 251 Mercer Street, New York, NY 10012, USA (masmoudi@cims.nyu.edu).

Nader Masmoudi was partially supported by NSF grant DMS-0703145.
} 
It is worth noting that there are many open problems related to the passage to the limit in the non linear terms due to the presence of boundary layers.

We study the following linearized compressible Navier-Stokes equations in a porous medium:

$$
\left\{\begin{aligned}
\partial_{t} p^{\varepsilon}+\operatorname{div} u^{\varepsilon} & =0 \quad \text { in }(0, \infty) \times \mathbb{R}_{\varepsilon}^{d}, \\
\partial_{t} u^{\varepsilon}-\varepsilon^{\beta} \triangle u^{\varepsilon}+\nabla p^{\varepsilon} & =0 \\
u^{\varepsilon} & =0 \quad \\
u^{\varepsilon}(t=0) & =a^{\varepsilon}, \\
p^{\varepsilon}(t=0) & =b^{\varepsilon}
\end{aligned}\right.
$$

where $p^{\varepsilon}$ and $u^{\varepsilon}$ are the pressure and velocity, and $\varepsilon^{\beta}$ is the viscosity. Here, $\mathbb{R}_{\varepsilon}^{d}$ is the porous medium formed by periodic repetition of an elementary fluid cell which has been shrunk to size $\varepsilon$ (see Section 2.1). Finally, $\beta \in \mathbb{R}$ measures the relative importance of the viscous effect to the size of each cell. As we will see in the appendix $\varepsilon^{\beta}$ and $\varepsilon$ are dimensional parameters and hence it makes sense to compare them. For a derivation of this model, we refer the reader to the Appendix where a dimensional analysis is performed.

Due to the possible vanishing of the viscosity at the limit, we can get the formation of boundary layers in space. In our problem, there are two boundary layers in space of size $\varepsilon^{(\beta+1) / 2}$ and $\varepsilon^{\beta / 2}$ corresponding to the incompressible and acoustic parts of the flow, respectively. Since the size of a cell is $\varepsilon$, we can only see a boundary layer if $\beta>1$. The value $\beta=1$ is a critical value and we define strongly viscous flow as the case when $\beta \leq 1$, i.e. the case when there is no boundary layer in space. This paper is an in-depth study of the strongly viscous regime.

Our goal in the paper is to describe the energy carried by the high frequency flow as the $\varepsilon$ goes to zero. Mathematically, we wish to understand the local and total energies

$$
\begin{aligned}
e^{\varepsilon}(t, x) & =\left|p^{\varepsilon}(t, x)\right|^{2}+\left|u^{\varepsilon}(t, x)\right|^{2}, \\
E^{\varepsilon}(t) & =\left\|p^{\varepsilon}(t)\right\|_{L^{2}\left(\mathbb{R}_{\varepsilon}^{d}\right)}^{2}+\left\|u^{\varepsilon}(t)\right\|_{L^{2}\left(\mathbb{R}_{\varepsilon}^{d}\right)}^{2}
\end{aligned}
$$

when fluid flow occurs in a porous medium, and when the limit $\varepsilon$ going to zero is taken. We present a complete description of the energy, which is motivated by formal two-scale expansions (see [18]).

Due to the viscous effects, the formation of boundary layers in time occurs which traps the flow during the usual two-scale convergence method. In the case $\beta \leq 0$, the usual formal two-scale expansions will describe the pressure, but will fail to describe the velocity for all times. Here, what we mean by the usual formal two-scale expansion is expressing $\left(u^{\varepsilon}, p^{\varepsilon}\right)$ as a formal expansion depending on $(t, x, y)$, where $y=x / \varepsilon$. These expansions show that the pressure equals its initial value on the microscopic scale when $\beta<0$, and is governed by the fluid flow Equation (2.7) when $\beta=0$. On the other hand, due to very strong viscous effects the usual two-scale expansions describe the velocity as zero. This failure to precisely describe the velocity is due to the formation of a boundary layer in time of size $\varepsilon^{2-\beta}$, which decouples the flow when $\varepsilon$ goes to zero. To describe the velocity near initial time, we need to introduce a different time scale during the homogenization process. Through this time rescaling, we deduce that the velocity is governed by heat flow near initial time (see Subsection 2.4 for formal arguments for the case $\beta=0$ ). The precise partition of the flow near initial time for 


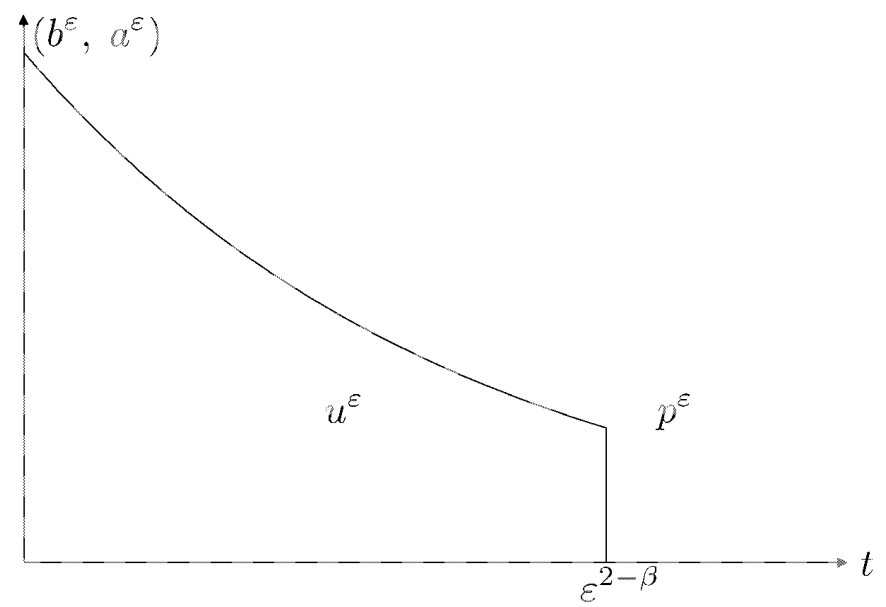

FIG. 1.1. The behavior of the flow near initial time for $\beta \leq 0$.

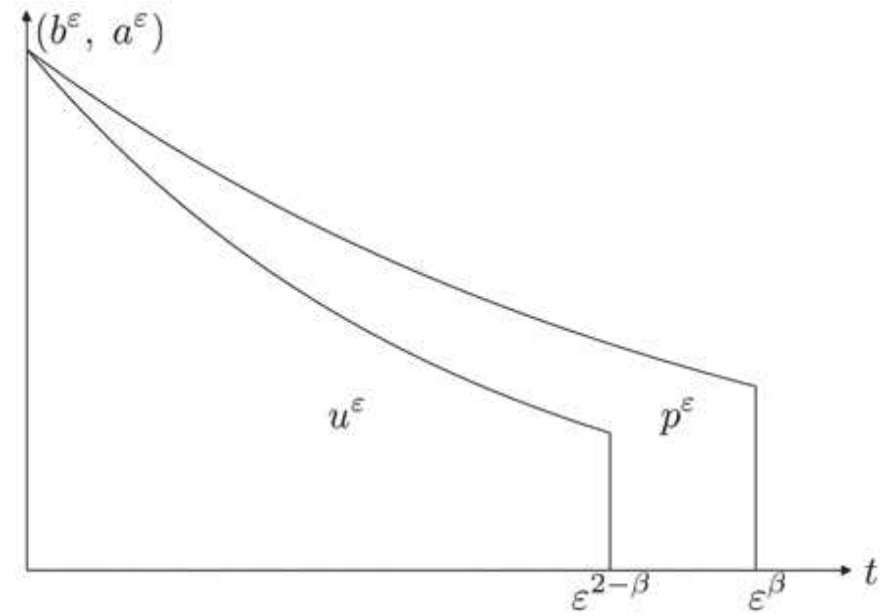

FIG. 1.2. The behavior of the flow near initial time for $0<\beta<1$.

$\beta \leq 0$ can be seen in Figure 1.1. In the case $\beta \in(0,1)$, the usual formal two-scale expansions will fail to capture the microscopic behavior of both the velocity and the pressure. This is due to the formation of initial layers in time of size $\varepsilon^{2-\beta}$ and $\varepsilon^{\beta}$ that trap the velocity and pressure, respectively. To capture the flow near initial time, we need to account for these layers in time by introducing appropriate time scales in the asymptotic expansions of the velocity and pressure. In doing so, we deduce that the microscopic limit equation governing the velocity and the pressure remains decoupled.

At the critical value $\beta=1$, the initial layers in time merge and the microscopic description of the pressure and velocity near initial time becomes coupled. In particular, we rescale time identically for both the pressure and velocity to describe the behavior of the flow near initial time.

We conclude with few remarks about the case $\beta>1$, i.e. the time-space boundary 


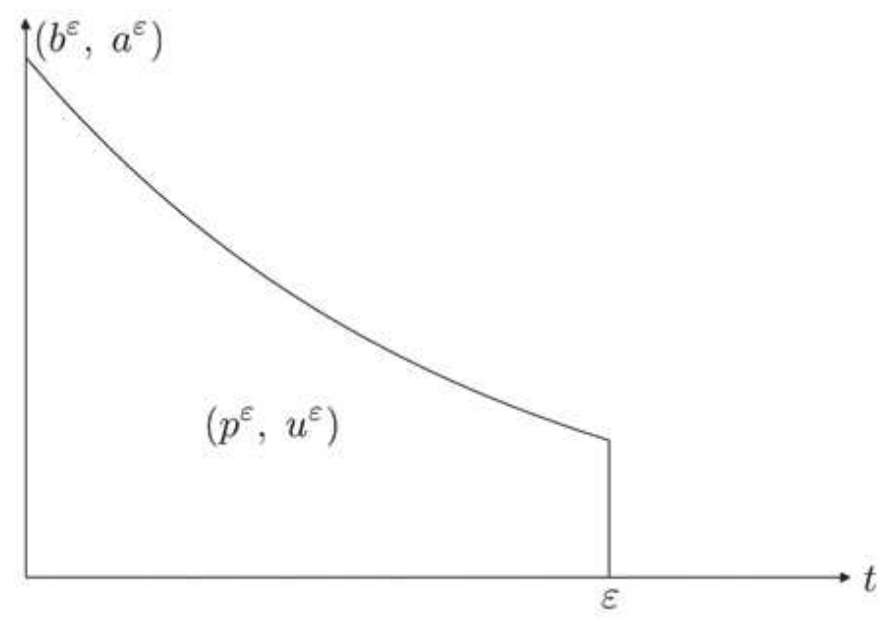

FIG. 1.3. The behavior of the flow near initial time for $\beta=1$.

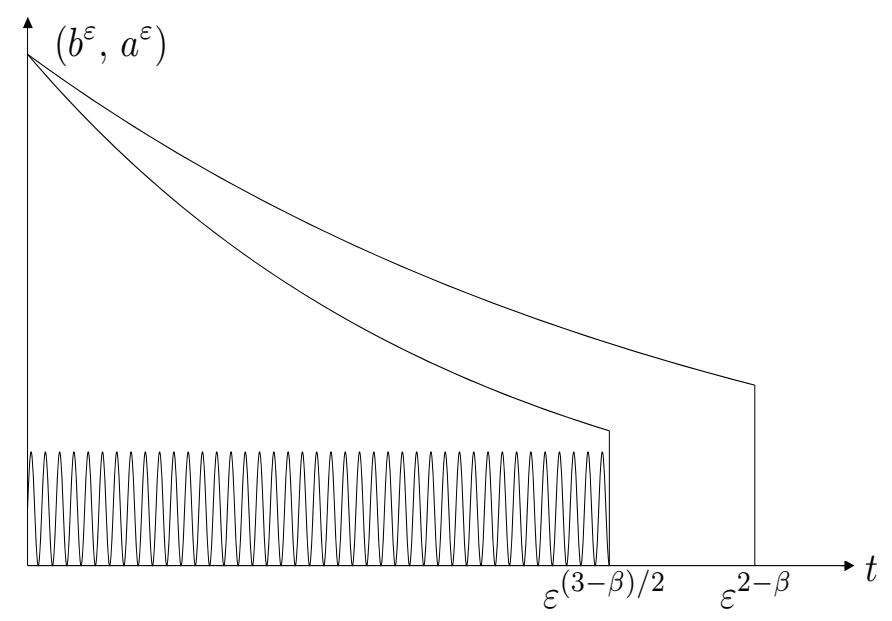

FIG. 1.4. Boundary layers in time for $1<\beta<2$.

layer problem. In the case of strongly viscous flow, we saw that the very strong viscous effects in space impacted the behavior of the flow near initial time and was responsible for the boundary layers in time. When $\beta>1$, we can ask the question: "How does the presence of boundary layers in space affect the flow near initial time?". We show that the boundary layer in time of size $\varepsilon^{2-\beta}$ remains from the case of strongly viscous flow, and that the boundary layer in space of size $\varepsilon^{(\beta+1) / 2}$ creates time oscillations of frequency $1 / \varepsilon$ and an initial layer in time of size $\varepsilon^{(3-\beta) / 2}$.

Note that when $\beta=1$, all the layers in time merge (see Figure 1.3). When $1<\beta<3$, time oscillations remain trapped and are damped, while time oscillations propagate forever when $\beta>3$. The value $\beta=3$ is a critical value, and we define mildly viscous flow as the case $1<\beta<3$ and weakly viscous flow as the case $\beta \geq 3$. We note that essentially 
there is another critical value, namely $\beta=2$, that corresponds to the disappearance of the initial layer in time of size $\varepsilon^{(2-\beta)}$ and boundary layer in space $\varepsilon^{\beta / 2}$. We do not define additional regimes corresponding to the case $\beta=2$.

Our forthcoming papers deal with the time-space boundary layer problem. In particular, we describe how these layers in time partition the flow and how the flow carries the energy in the homogenization process in the case of $\beta>1$. More precisely, we show that the layer in time of size $\varepsilon^{2-\beta}$ captures the micro-incompressible part of the flow, i.e. the part of the flow that is divergence free on the microscopic level, i.e. $\operatorname{div}_{y} u(x, y)=0$. A detailed study of the energy carried by micro-incompressible flow, in case $\beta>1$, is carried out in our second paper [7]. Our final paper, [6], is a complete study of micro-acoustic flow in the mild and weakly viscous regimes. Here micro-acoustic flow is defined to be the part of the flow trapped by the layer in time $\varepsilon^{(3-\beta) / 2}$. Notice that micro-acoustic flow oscillates in time at the frequency $1 / \varepsilon$.

In conclusion, we remark on the various weak convergence tools needed to deal with the extra difficulty of time-space boundary layers. In addition to two-scale convergence, the Bloch decomposition and the Wigner measure are applied. To emphasize the need for tools beyond two-scale convergence, we refer the reader to Section 5 for formal arguments in the case $\beta=2$ and to compare this with Section 2.4.

\section{Preliminaries}

2.1. The Domain. Define $Y=(0,1)^{d}$ to be the unit open cube in $\mathbb{R}^{d}$, and let $Y_{s}$ be a closed smooth subset of $Y$ with strictly positive measure. By smoothness of $\partial Y_{s}$, we mean to take it as regular as needed. The domain $Y / Y_{s}$ is denoted by $Y_{f}$ and we refer to $Y_{s}, Y_{f}$ to be the solid and fluid parts of $Y$. Repeating $Y_{f}$ by $Y$-periodicity to all of $\mathbb{R}^{d}$ we get the fluid domain $\mathbb{R}_{0}$. The porous medium is now defined to be $\varepsilon \mathbb{R}_{0}$, which is denoted by $\mathbb{R}_{\varepsilon}^{d}$. We define $Y_{f}^{\varepsilon}, Y_{s}^{\varepsilon}$ to be $\varepsilon Y_{f}, \varepsilon Y_{s}$ and note that

$$
\mathbb{R}_{\varepsilon}^{d}=\left\{x: x \in Y_{f}^{\varepsilon}+p \text {, for some } p \in \mathbb{Z}^{d}\right\} .
$$

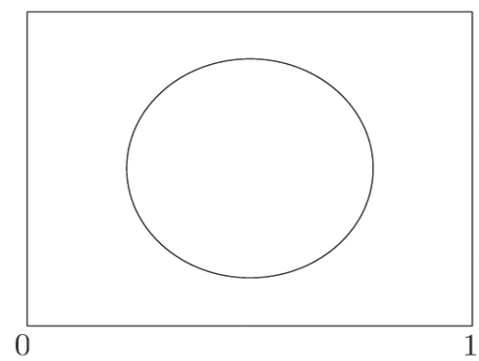

FIG. 2.1. The elementary fluid cell.

2.2. Notations. Throughout this paper, we define $C_{\#}^{\infty}(Y)$ to be the space of all infinitely differentiable functions on $\mathbb{R}^{d}$ which are $Y$-periodic. The space of functions which are $C^{\infty}$ on $\mathbb{R}^{d}$, and have compact support, are denoted by $D\left(\mathbb{R}^{d}\right)$. We define the space $L_{\#}^{2}\left(Y_{f}\right)$ to be all functions belonging to $L_{l o c}^{2}\left(\mathbb{R}_{0}^{d}\right)$ which are $Y$ periodic, and $H_{\#}^{k}\left(Y_{f}\right)$ to be the space of functions with derivatives up to order $k$ 
belonging to $L_{\#}^{2}\left(Y_{f}\right)$. When $k=1$, the space $H_{0 \#}^{1}\left(Y_{f}\right)$ is defined to be all functions belonging to $H_{\#}^{1}\left(Y_{f}\right)$ which vanish on $\partial Y_{s}$. We define the scalar products

$$
(u, v)_{L_{\#}^{2}\left(Y_{f}\right)}=\int_{Y_{f}} u v d x, \quad(u, v)_{H_{0 \#}^{1}\left(Y_{f}\right)}=\int_{Y_{f}} \nabla u \cdot \nabla v d x
$$

and also recall that the space $H_{\#}^{-1}\left(Y_{f}\right)$ is defined by the norm

$$
\|f\|_{H_{\#}^{-1}\left(Y_{f}\right)}=\sup _{\|u\|_{H_{0 \#}^{1}\left(Y_{f}\right)}=1}<f, u>.
$$

Also, when no confusion is possible, the symbol \# will be omitted in my formulas.

We conclude by defining $X^{d}$ as the Banach space of vector valued functions of d-components, each component belonging to the Banach space $X$.

2.3. Two-scale convergence. To write the two-scale homogenized equation we need the notion of two-scale convergence. This notion was first introduced by G. Nguetseng [16, 17] and later extended by G. Allaire [2]. In the sequel, we will use two-scale convergence to capture the part of $f^{\varepsilon}$ which oscillates at frequency $\varepsilon^{-1}$ in the $x$-variable only. In our problem two-scale convergence plays a significant role in understanding the local and total energies (1.2).

Definition 2.1. Let $f^{\varepsilon}(x), f_{0}(x, y)$ belong to $L^{2}\left(\mathbb{R}_{\varepsilon}^{d}\right), L^{2}\left(\mathbb{R}^{d} \times Y_{f}\right)$ respectively. We say that $f^{\varepsilon}(x)$ two-scale converges to $f_{0}(x, y)$ (denoted $f^{\varepsilon} \stackrel{2-s}{\longrightarrow} f_{0}$ ) provided

$$
\int_{\mathbb{R}_{\varepsilon}^{d}} f^{\varepsilon}(x) \sigma\left(x, \frac{x}{\varepsilon}\right) \stackrel{\varepsilon \rightarrow 0}{\rightarrow} \int_{\mathbb{R}^{d}} \int_{Y_{f}} f_{0}(x, y) \sigma(x, y)
$$

for all $\sigma \in D\left(\mathbb{R}^{d} ; C_{\#}^{\infty}\left(Y_{f}\right)\right)$.

Definition 2.2. We say $f^{\varepsilon}$ strongly two-scale converges to $f_{0}(x, y)$, denoted $f^{\varepsilon} \stackrel{2-s}{\rightarrow}$ $f_{0}(x, y)$, provided $f^{\varepsilon}$ two-scale converges to $f_{0}$ and

$$
\lim _{\varepsilon \rightarrow 0}\left\|f^{\varepsilon}\right\|_{L^{2}\left(\mathbb{R}_{\varepsilon}^{d}\right)}^{2}=\left\|f_{0}\right\|_{L^{2}\left(\mathbb{R}^{d} \times Y_{f}\right)}^{2} .
$$

REMARK 2.1. Notice that if $f^{\varepsilon}(x)$ two-scale converges to $f_{0}(x, y)$ then the following always holds:

$$
\lim _{\varepsilon \rightarrow 0}\left\|f^{\varepsilon}\right\|_{L^{2}\left(\mathbb{R}_{\varepsilon}^{d}\right)}^{2} \geq\left\|f_{0}\right\|_{L^{2}\left(\mathbb{R}^{d} \times Y_{f}\right)}^{2} .
$$

The inequality follows from Definition 2.1, where we only capture the oscillations of the sequence $f^{\varepsilon}$ in resonance with the test function $\sigma$.

Strong two-scale convergence is interpreted as saying all of the oscillations of $f^{\varepsilon}$ are of frequency $\varepsilon^{-1}$. This is due to the fact that strong two-scale convergence implies

$$
\lim _{\varepsilon \rightarrow 0}\left\|f^{\varepsilon}\right\|_{L^{2}\left(\mathbb{R}_{\varepsilon}^{d}\right)}=\lim _{\varepsilon \rightarrow 0}\left\|f_{0}\left(x, \frac{x}{\varepsilon}\right)\right\|_{L^{2}\left(\mathbb{R}_{\varepsilon}^{d}\right)}
$$

whenever $f_{0}$ is continuous in either the $x$ or $y$ variable. More generally, (2.3) holds provided $f_{0}$ is an admissible test function in the sense of Allaire (see Definition 1.4 of [2]). We refer to Proposition 2.3 for further discussion on the characterization of strong two-scale convergence. We note that given $f_{0}(x, y)$, having continuity in at 
least one of its variables enables us to make sense of the quantity $f_{0}\left(x, \frac{x}{\varepsilon}\right)$. Indeed, this follows from the fact that $f_{0}\left(x, \frac{x}{\varepsilon}\right)$ is the trace of the function $f_{0}(x, y)$. We refer the reader to Section 5 of [2] for further discussion. If $f^{\varepsilon}$ strong two-scale converges to $f_{0} \in L^{2}\left(\mathbb{R}^{d} \times Y_{f}\right)$ and if, in addition, $f_{0}(x, y)$ is an admissible test function, then we can improve the convergence of (2.3). Indeed, we have the following:

$$
\lim _{\varepsilon \rightarrow 0} \int_{\mathbb{R}_{\varepsilon}^{d}}\left|f^{\varepsilon}(x)-f_{0}\left(x, \frac{x}{\varepsilon}\right)\right|^{2}=0 .
$$

Finally, we can add a time dependence in all the limits we mentioned above. In particular if $f_{0} \in C\left([0, T] ; L_{\#}^{2}\left(Y_{f} ; C\left(\mathbb{R}^{d}\right) \cap L^{2}\left(\mathbb{R}^{d}\right)\right)\right)$, then we have

$$
\lim _{\varepsilon \rightarrow 0}\left\|f_{0}\left(t, x, \frac{x}{\varepsilon}\right)\right\|_{L^{2}\left((0, T) \times \mathbb{R}_{\varepsilon}^{d}\right)}=\left\|f_{0}\right\|_{L^{2}\left((0, T) \times \mathbb{R}^{d} \times Y_{f}\right)} .
$$

We now state an existence theorem regarding the two-scale limit for bounded sequences in $L^{2}\left(\mathbb{R}_{\varepsilon}^{d}\right)$. For a proof, we refer the reader to [16] and [2].

TheOREM 2.2. Let $f^{\varepsilon}$ belong to a bounded set of $L^{2}\left(\mathbb{R}_{\varepsilon}^{d}\right)$. Then there exist a subsequence of $\varepsilon$ (still denoted by $\varepsilon$ ) and a $Y$-periodic function $f_{0}(x, y) \in L^{2}\left(\mathbb{R}^{d} \times Y_{f}\right)$, such that $f^{\varepsilon}(x)$ two-scale converges to $f_{0}(x, y)$.

We conclude by stating the following Proposition which characterizes the strong two-scale limit. We first introduce the following mollifier in the $x$ variable. Let $\omega \in C_{0}^{\infty}\left(\mathbb{R}^{d}\right)$ be such that $\omega \geq 0, \int \omega=1$, and $\operatorname{Supp}(\omega) \in B(0,1)$. We denote by $f \star$ $\omega_{\delta}(x, y)=\int f(x-z, y) \omega_{\delta}(z) d z$ the convolution in the $x$ variable of $f$, where $\omega_{\delta}(x)=$ $\frac{1}{\delta^{d}} \omega\left(\frac{x}{\delta}\right)$.

Proposition 2.3. Assume that $f^{\varepsilon}$ is bounded in $L^{2}\left(\mathbb{R}_{\varepsilon}^{d}\right)$ and two-scale converges to a function $f_{0}(x, y) \in L^{2}\left(\mathbb{R}^{d} \times Y_{f}\right)$. Then $f^{\varepsilon}$ two-scale converges strongly to $f_{0}(x, y)$ if and only if the following holds:

$$
\lim _{\delta \rightarrow 0} \limsup _{\varepsilon \rightarrow 0}\left\|f^{\varepsilon}(x)-f_{0} \star \omega_{\delta}\left(x, \frac{x}{\varepsilon}\right)\right\|_{L^{2}\left(\mathbb{R}^{d}\right)}=0 .
$$

Proof. Assume that $f^{\varepsilon}$ two-scale converges strongly to $f_{0}(x, y)$. To prove (2.5), we use that $f_{0} \star \omega_{\delta}\left(x, \frac{x}{\varepsilon}\right)$ two-scale converges (and even two-scale converges strongly) to $f_{0} \star \omega_{\delta}(x, y)$ and hence, by Theorem 1.8 of [2], we deduce that

$$
\lim _{\varepsilon \rightarrow 0} \int_{\mathbb{R}_{\varepsilon}^{d}} f^{\varepsilon}(x) f_{0} \star \omega_{\delta}\left(x, \frac{x}{\varepsilon}\right)=\int_{\mathbb{R}^{d} \times Y_{f}} f_{0}(x, y) f_{0} \star \omega_{\delta}(x, y),
$$

and hence

$$
\lim _{\varepsilon \rightarrow 0}\left\|f^{\varepsilon}(x)-f_{0} \star \omega_{\delta}\left(x, \frac{x}{\varepsilon}\right)\right\|_{L^{2}\left(\mathbb{R}_{\varepsilon}^{d}\right)}=\left\|f_{0}(x, y)-f_{0} \star \omega_{\delta}(x, y)\right\|_{L^{2}\left(\mathbb{R}^{d} \times Y_{f}\right)} .
$$

Sending $\delta$ to zero, we deduce that (2.5) holds.

Conversely, if (2.5) holds, then for $\alpha>0$ there exists $\delta_{0}$ such that for $\delta<\delta_{0}$, we have

$$
\lim _{\varepsilon \rightarrow 0}\left\|f^{\varepsilon}(x)-f_{0} \star \omega_{\delta}\left(x, \frac{x}{\varepsilon}\right)\right\|_{L^{2}\left(\mathbb{R}_{\varepsilon}^{d}\right)}<\alpha
$$


Also, there exists $\delta_{1}$ such that for $\delta<\delta_{1}$, we have

$$
\left\|f_{0}(x, y)-f_{0} \star \omega_{\delta}\left(x, \frac{x}{\varepsilon}\right)\right\|_{L^{2}\left(\mathbb{R}^{d} \times Y_{f}\right)}<\alpha .
$$

Taking $\delta<\min \left(\delta_{0}, \delta_{1}\right)$, we deduce that

$$
\left|\lim _{\varepsilon \rightarrow 0}\left\|f^{\varepsilon}(x)\right\|_{L^{2}\left(\mathbb{R}_{\varepsilon}^{d}\right)}-\left\|f_{0}(x, y)\right\|_{L^{2}\left(\mathbb{R}^{d} \times Y_{f}\right)}\right| \leq C \alpha,
$$

and hence (2.2) holds.

2.4. Formal Asymptotics for $\beta=0$. Before stating the main results, we present a formal argument for the case $\beta=0$. This formal process motivates all the conclusions of this paper, and we refer the reader to [5] for the formal arguments for other values of $\beta$.

In the usual manner one assumes that $\left(p^{\varepsilon}, u^{\varepsilon}\right)$ admits the following two-scale expansion (see [18]):

$$
\begin{aligned}
& p^{\varepsilon}=\sum_{j=0}^{\infty} \varepsilon^{j} p_{j}\left(t, x, \frac{x}{\varepsilon}\right), \\
& u^{\varepsilon}=\sum_{j=0}^{\infty} \varepsilon^{j} u_{j}\left(t, x, \frac{x}{\varepsilon}\right),
\end{aligned}
$$

where the functions $\left(p_{j}, u_{j}\right)$ are periodic in $y$. Upon doing so, we conclude that $\triangle_{y} u_{0}=0$ and the following two-scale system for $\left(p_{0}, u_{1}\right)$ :

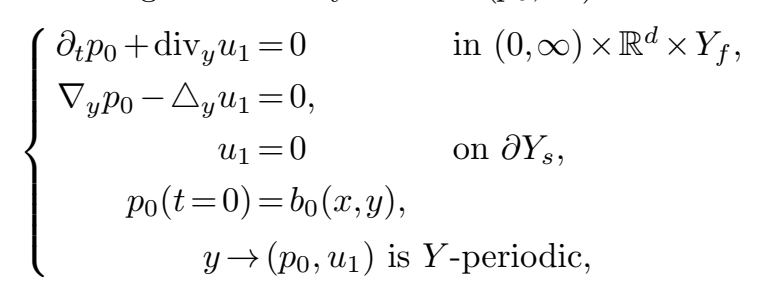

where $b_{0}(x, y)$ is the two-scale limit of $b^{\varepsilon}$.

We conclude immediately that two-scale convergence (without time rescaling) captures the part of the flow with initial condition $\left(b^{\varepsilon}, 0\right)$. Furthermore, the part of the flow with initial value $\left(0, a^{\varepsilon}\right)$ is captured using the following expansions where time is also rescaled:

$$
\begin{aligned}
& p^{\varepsilon}=\sum_{j=0}^{\infty} \varepsilon^{j} p_{j}\left(\frac{t}{\varepsilon^{2}}, x, \frac{x}{\varepsilon}\right), \\
& u^{\varepsilon}=\sum_{j=0}^{\infty} \varepsilon^{j} u_{j}\left(\frac{t}{\varepsilon^{2}}, x, \frac{x}{\varepsilon}\right) .
\end{aligned}
$$

We point out that we are using the same notation $u_{0}, u_{1}, \ldots$ and $p_{0}, p_{1}, \ldots$ as in (2.6). There will be no confusion later since $\left(u_{1}, p_{0}\right)$ and $u_{0}$, used to describe the pressure and velocity $p^{\varepsilon}, u^{\varepsilon}$, have different time scales. Notice that here, the main part of flow near initial time is described by the following heat equation:

$$
\left\{\begin{array}{rlrl}
\partial_{\tau} u_{0}-\triangle_{y} u_{0} & =0 & & \text { in }(0, \infty) \times \mathbb{R}^{d} \times Y_{f}, \\
u_{0} & =0 & & \text { on } \partial Y_{s}, \\
u_{0}(\tau=0) & =a_{0}(x, y), & \\
y & \rightarrow u_{0} \text { is } Y \text {-periodic. }
\end{array}\right.
$$


Here $\tau=t / \varepsilon^{2}$ and $a_{0}(x, y)$ is the two-scale limit of $a^{\varepsilon}$.

System (2.8) shows that the velocity $u_{0}$ is dominated by the viscous effects that yield the damping of $u_{0}$ on a very short time scale. Besides, system (2.7) shows that the dissipation of $p_{0}$ happens on a time scale of order 1 and is due to the second order velocity $u_{1}$.

\section{Statement of the main results}

In order to state the main results, we impose the following conditions on the initial data $\left(b^{\varepsilon}, a^{\varepsilon}\right)$. We assume that

(A1) $\left(b^{\varepsilon}, a^{\varepsilon}\right)^{2-s} \rightarrow\left(b_{0}(x, y), a_{0}(x, y)\right)$.

We now state the main results.

THEOREM 3.1. $(\beta<0)$ Let $(\boldsymbol{A} 1)$ hold and assume the following regularity:

$$
\partial_{x}^{\alpha} a_{0} \in L^{2}\left(\mathbb{R}^{d} \times Y_{f}\right), \quad \forall|\alpha| \leq 2 .
$$

Let $\left(p^{\varepsilon}, u^{\varepsilon}\right) \in C\left([0, T) ; L^{2}\left(\mathbb{R}_{\varepsilon}^{d}\right) \times L^{2}\left(\mathbb{R}_{\varepsilon}^{d}\right)^{d}\right)$ be the solution to (1.1) for $\beta<0$ and assume we are in dimensions $d=2,3$. In addition, assume $b_{0}$ is an admissible test function (see Remark 2.1). Then for $T \in(0, \infty)$, we have the following description of the energy:

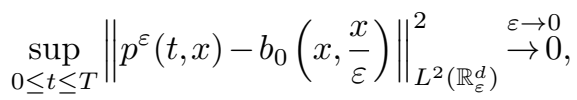

$$
\begin{aligned}
& \sup _{0 \leq t \leq T}\left\|u^{\varepsilon}(t, x)-u_{0}\left(\frac{t}{\varepsilon^{2-\beta}}, x, \frac{x}{\varepsilon}\right)\right\|_{L^{2}\left(\mathbb{R}_{\varepsilon}^{d}\right)}^{2} \stackrel{\varepsilon \rightarrow 0}{\rightarrow 0}, \\
& \varepsilon^{\beta} \int_{0}^{T}\left\|\nabla u^{\varepsilon}(t, x)-\frac{1}{\varepsilon} \nabla_{y} u_{0}\left(\frac{t}{\varepsilon^{2-\beta}}, x, \frac{x}{\varepsilon}\right)\right\|_{L^{2}\left(\mathbb{R}_{\varepsilon}^{d}\right)}^{2} \stackrel{\substack{\varepsilon \rightarrow 0 \\
\rightarrow 0}}{2}
\end{aligned}
$$

where $u_{0}$ satisfies (2.8).

Notice in particular that for this case the pressure strongly two-scale converges, uniformly in time, to its initial data.

Now, we turn to the case $0 \leq \beta<1$. Motivated by the formal argument of Section 2.4 , we notice that in order to rigorously describe the energy carried by the pressure $p^{\varepsilon}$, we need to deduce existence as well as general properties for the microscopic system (2.7), which seems to be interesting by itself. We have the following result:

Theorem 3.2. $(0 \leq \beta<1$, Existence for the limit system (2.7))

(i) Let $b_{0}(x, \cdot) \in L^{2}\left(Y_{f}\right)$. Then there exists a unique solution $\left(p_{0}, u_{1}\right)(t, x, \cdot) \in$ $C\left([0, T) ; L^{2}\left(Y_{f}\right) \times H_{0 \#}^{1}\left(Y_{f}\right)^{d}\right)$ satisfying system (2.7). In addition, $\left(p_{0}, u_{1}\right)$ satisfy the following energy equalities:

$$
\begin{gathered}
\left\|p_{0}(t, x, \cdot)\right\|_{L^{2}\left(Y_{f}\right)}^{2}+\int_{0}^{t}\left\|\nabla_{y} u_{1}(s, x, \cdot)\right\|_{L^{2}\left(Y_{f}\right)}^{2}=\left\|b_{0}(x, \cdot)\right\|_{L^{2}\left(Y_{f}\right)}^{2}, \\
\left\|\nabla_{y} u_{1}(s, x, \cdot)\right\|_{L^{2}\left(Y_{f}\right)}^{2}+\int_{0}^{t}\left\|d i v_{y} u_{1}(s, x, \cdot)\right\|_{L^{2}\left(Y_{f}\right)}^{2}=\left\|\nabla \triangle_{D}^{-1} \nabla b_{0}(x, \cdot)\right\|_{L^{2}\left(Y_{f}\right)}^{2} .
\end{gathered}
$$

(ii) The solution constructed in (i) satisfies $\partial_{t}^{k}\left(p_{0}, u_{1}\right)(\cdot, x, \cdot) \in L^{2}\left((0, \infty) \times Y_{f}\right) \times$ $L^{2}\left(0, \infty ; H_{0 \#}^{1}\left(Y_{f}\right)\right)^{d}$ for all $k \in \mathbb{N}$ and satisfies the bound

$$
\left\|\partial_{t}^{k} p_{0}(\cdot, x, \cdot)\right\|_{L^{2}\left((0, \infty) \times Y_{f}\right)}+\left\|\partial_{t}^{k} u_{1}(\cdot, x, \cdot)\right\|_{L^{2}\left((0, \infty) \times Y_{f}\right)} \leq C\left\|b_{0}(x, \cdot)\right\|_{L^{2}\left(Y_{f}\right)}
$$


(iii) Moreover, we have

$$
\begin{array}{r}
\left\|\left(p_{0}(t, x, \cdot)-f_{Y_{f}} b_{0}(x, y)\right)\right\|_{L^{2}\left(Y_{f}\right)} \leq C_{0}\left\|b_{0}(x, \cdot)\right\|_{L^{2}\left(Y_{f}\right)} e^{-C t} \\
\left\|u_{1}(t, x, \cdot)\right\|_{H_{0}^{1}\left(Y_{f}\right)} \leq C_{0}\left\|b_{0}(x, \cdot)\right\|_{L^{2}\left(Y_{f}\right)} e^{-C t} .
\end{array}
$$

The asymptotic behavior of the pressure and velocity obtained for $\beta=0$ in the formal arguments of Section 2.4 carries over to the case $\beta \in[0,1)$. The precise theorem reads as follows:

THEOREM 3.3. $(0 \leq \beta<1)$ Let $(\boldsymbol{A} 1)$ hold and assume the following regularity:

$$
\partial_{x}^{\alpha} b_{0} \in L^{2}\left(\mathbb{R}^{d} ; L_{\#}^{2}\left(Y_{f}\right)\right), \quad \partial_{x}^{\alpha} a_{0} \in L^{2}\left(\mathbb{R}^{d} ; L_{\#}^{2}\left(Y_{f}\right)\right), \quad \forall|\alpha| \leq 4 .
$$

Let $\left(p^{\varepsilon}, u^{\varepsilon}\right) \in C\left([0, T) ; L^{2}\left(\mathbb{R}_{\varepsilon}^{d}\right) \times L^{2}\left(\mathbb{R}_{\varepsilon}^{d}\right)^{d}\right)$ be the unique solution to (1.1) for $\beta \in[0,1)$ and assume we are in dimensions $d=2,3$. Then for $T \in(0, \infty)$, we have the following description of the energy:

$$
\begin{aligned}
& \sup _{0 \leq t \leq T}\left\|p^{\varepsilon}(t, x)-p_{0}\left(\frac{t}{\varepsilon^{\beta}}, x, \frac{x}{\varepsilon}\right)\right\|_{L^{2}\left(\mathbb{R}_{\varepsilon}^{d}\right)}^{2} \stackrel{\varepsilon \rightarrow 0}{\rightarrow 0} \\
& \sup _{0 \leq t \leq T}\left\|u^{\varepsilon}(t, x)-u_{0}\left(\frac{t}{\varepsilon^{2-\beta}}, x, \frac{x}{\varepsilon}\right)\right\|_{L^{2}\left(\mathbb{R}_{\varepsilon}^{d}\right)}^{2} \stackrel{\varepsilon \rightarrow 0}{\rightarrow 0},
\end{aligned}
$$

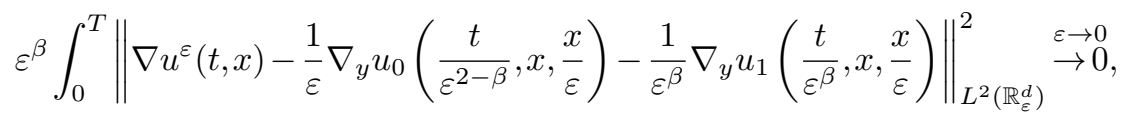

where $\left(p_{0}, u_{1}\right)$ and $u_{0}$ satisfy the systems (2.7) and (2.8) respectively.

REMARK 3.4. Notice that the flow remains uncoupled for $\beta<1$ (see Figure 1.1, 1.2).

We conclude with the results concerning the critical value $\beta=1$. When the layers in time merge, the microscopic description of the pressure and velocity becomes coupled (see Figure 1.3). The main result reads

TheOrem 3.5. $(\beta=1)$ Let (A1) hold and assume the following regularity:

$$
\partial_{x}^{\alpha} b_{0} \in L^{2}\left(\mathbb{R}^{d} ; L^{2}\left(Y_{f}\right)\right), \quad \partial_{x}^{\alpha} a_{0} \in L^{2}\left(\mathbb{R}^{d} ; L^{2}\left(Y_{f}\right)\right), \quad \forall|\alpha| \leq 4 .
$$

Let $\left(p^{\varepsilon}, u^{\varepsilon}\right) \in C\left([0, T) ; L^{2}\left(\mathbb{R}_{\varepsilon}^{d}\right) \times L^{2}\left(\mathbb{R}_{\varepsilon}^{d}\right)^{d}\right)$ be the solution to (1.1) for $\beta=1$, and assume we are in dimensions $d=2,3$. Then for $T \in(0, \infty)$, we have the following description of the energy:

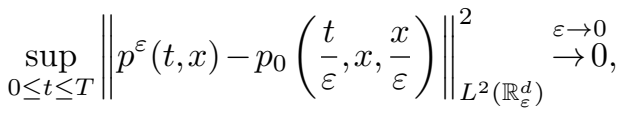

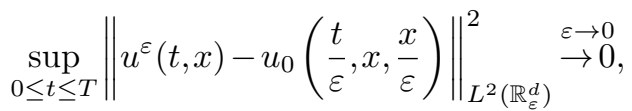

$$
\begin{aligned}
& \varepsilon \int_{0}^{T}\left\|\nabla u^{\varepsilon}(t, x)-\frac{1}{\varepsilon} \nabla_{y} u_{0}\left(\frac{t}{\varepsilon}, x, \frac{x}{\varepsilon}\right)\right\|_{2}^{2} \stackrel{\varepsilon \rightarrow 0}{\rightarrow} 0,
\end{aligned}
$$


where $\left(p_{0}, u_{0}\right) \in C\left([0, T) ; L^{2}\left(\mathbb{R}^{d}\right) \times L^{2}\left(\mathbb{R}^{d}\right)^{d}\right)$ is the unique solution of the following system:

$$
\left\{\begin{array}{rlrl}
\partial_{\tau} p_{0}+d i v_{y} u_{0} & =0 & & \\
\partial_{\tau} u_{0}-\triangle_{y} u_{0}+\nabla_{y} p_{0} & =0, & & \\
u_{0} & =0 & & \\
p_{0}(\tau=0) & =b_{0}(x, y), & & \\
u_{0}(\tau=0) & =a_{0}(x, y), & & \\
y \rightarrow p_{0}, & u_{0} & \text { is } Y \text {-periodic. }
\end{array}\right.
$$

REMARK 3.6.

1) Notice that energy is dissipated by the heat flow in the case $\beta<0$. As our fluid becomes less viscous, i.e. $\beta \rightarrow 1-$, the intensity with which the energy is dissipated weakens.

2) In all the systems we obtained for $\beta \leq 1$, the variable $x$ appears as a parameter. There is no macroscopic flow (flow in the $x$ variable). This is due to the fact that the viscosity is very strong.

3) In the three convergence Theorems we stated, we did not try to give a rate of convergence in terms of $\varepsilon$. One can easily get some error estimates in terms of $\varepsilon$ by computing the next terms in the formal asymptotic expansions, but this is not our goal in this paper.

4) Also, we did not try to get the best possible regularity on the initial data and one can easily lower the regularity requirement on the initial data. Indeed, the regularity in $x$ of the initial data is used to control the force terms $F^{\varepsilon}$ and $G^{\varepsilon}$, which loose derivatives (see Section 4), and also to be able to define the trace on the line $\left(x, \frac{x}{\varepsilon}\right)$ of some functions. We will state corollaries where the regularity requirements are completely removed but we have to use a mollification in the $x$ variable to state our convergence result.

Corollary 3.7. $(\beta<0)$ Let $(\boldsymbol{A} 1)$ hold and let $\left(p^{\varepsilon}, u^{\varepsilon}\right) \in C\left([0, T) ; L^{2}\left(\mathbb{R}_{\varepsilon}^{d}\right) \times L^{2}\left(\mathbb{R}_{\varepsilon}^{d}\right)^{d}\right)$ be the solution to (1.1) for $\beta<0$. Then for $T \in(0, \infty)$, we have the following description of the energy:

$$
\begin{aligned}
& \lim _{\delta \rightarrow 0} \limsup _{\varepsilon \rightarrow 0} \sup _{0 \leq t \leq T}\left\|p^{\varepsilon}(t, x)-b_{0} \star \omega_{\delta}\left(x, \frac{x}{\varepsilon}\right)\right\|_{L^{2}\left(\mathbb{R}_{\varepsilon}^{d}\right)}^{2}=0, \\
& \lim _{\delta \rightarrow 0} \limsup _{\varepsilon \rightarrow 0} \sup _{0 \leq t \leq T}\left\|u^{\varepsilon}(t, x)-u_{0} \star \omega_{\delta}\left(\frac{t}{\varepsilon^{2-\beta}}, x, \frac{x}{\varepsilon}\right)\right\|_{L^{2}\left(\mathbb{R}_{\varepsilon}^{d}\right)}^{2}=0, \\
& \lim _{\delta \rightarrow 0} \limsup _{\varepsilon \rightarrow 0} \varepsilon^{\beta} \int_{0}^{T}\left\|\nabla u^{\varepsilon}(t, x)-\frac{1}{\varepsilon} \nabla_{y} u_{0} \star \omega_{\delta}\left(\frac{t}{\varepsilon^{2-\beta}}, x, \frac{x}{\varepsilon}\right)\right\|_{L^{2}\left(\mathbb{R}_{\varepsilon}^{d}\right)}^{2}=0,
\end{aligned}
$$

where $u_{0}$ satisfies (2.8). If in addition $b_{0}$ is an admissible test function (in the sense of Definition 1.4 of [2]), then (3.1) still holds without the necessity of using a mollification. 
Corollary 3.8. $\quad(0 \leq \beta<1) \quad$ Let $(\boldsymbol{A} 1)$ hold and let $\left(p^{\varepsilon}, u^{\varepsilon}\right) \in C\left([0, T) ; L^{2}\left(\mathbb{R}_{\varepsilon}^{d}\right) \times\right.$ $\left.L^{2}\left(\mathbb{R}_{\varepsilon}^{d}\right)^{d}\right)$ be the unique solution to (1.1) for $\beta \in[0,1)$. Then for $T \in(0, \infty)$, we have the following description of the energy:

$$
\begin{gathered}
\lim _{\delta \rightarrow 0} \limsup _{\varepsilon \rightarrow 0} \sup _{0 \leq t \leq T}\left\|p^{\varepsilon}(t, x)-p_{0} \star \omega_{\delta}\left(\frac{t}{\varepsilon^{\beta}}, x, \frac{x}{\varepsilon}\right)\right\|_{L^{2}\left(\mathbb{R}_{\varepsilon}^{d}\right)}^{2}=0, \\
\lim _{\delta \rightarrow 0} \limsup _{\varepsilon \rightarrow 0} \sup _{0 \leq t \leq T}\left\|u^{\varepsilon}(t, x)-u_{0} \star \omega_{\delta}\left(\frac{t}{\varepsilon^{2-\beta}}, x, \frac{x}{\varepsilon}\right)\right\|_{L^{2}\left(\mathbb{R}_{\varepsilon}^{d}\right)}^{2}=0, \\
\lim _{\delta \rightarrow 0} \limsup _{\varepsilon \rightarrow 0} \varepsilon^{\beta} \int_{0}^{T} \| \nabla u^{\varepsilon}(t, x)-\frac{1}{\varepsilon} \nabla_{y} u_{0} \star \omega_{\delta}\left(\frac{t}{\varepsilon^{2-\beta}}, x, \frac{x}{\varepsilon}\right) \\
-\frac{1}{\varepsilon^{\beta}} \nabla_{y} u_{1} \star \omega_{\delta}\left(\frac{t}{\varepsilon^{\beta}}, x, \frac{x}{\varepsilon}\right) \|_{L^{2}\left(\mathbb{R}_{\varepsilon}^{d}\right)}^{2}=0,
\end{gathered}
$$

where $\left(p_{0}, u_{1}\right)$ and $u_{0}$ satisfy the systems (2.7) and (2.8) respectively. If in addition $\left(p_{0}, u_{1}\right)$ and $\nabla_{y} u_{1}$ are admissible test functions for each $t \in[0, T]$, then (3.2) still holds without the necessity of using a mollification.

Corollary 3.9. $(\beta=1)$ Let (A1) hold and let $\left(p^{\varepsilon}, u^{\varepsilon}\right)$ be the solution to (1.1) for $\beta=1$. Then for $T \in(0, \infty)$, we have the following description of the energy:

$$
\begin{aligned}
& \lim _{\delta \rightarrow 0} \limsup _{\varepsilon \rightarrow 0} \sup _{0 \leq t \leq T}\left\|p^{\varepsilon}(t, x)-p_{0} \star \omega_{\delta}\left(\frac{t}{\varepsilon}, x, \frac{x}{\varepsilon}\right)\right\|_{L^{2}\left(\mathbb{R}_{\varepsilon}^{d}\right)}^{2}=0, \\
& \lim _{\delta \rightarrow 0} \limsup _{\varepsilon \rightarrow 0} \sup _{0 \leq t \leq T}\left\|u^{\varepsilon}(t, x)-u_{0} \star \omega_{\delta}\left(\frac{t}{\varepsilon}, x, \frac{x}{\varepsilon}\right)\right\|_{L^{2}\left(\mathbb{R}_{\varepsilon}^{d}\right)}^{2}=0, \\
& \lim _{\delta \rightarrow 0} \limsup _{\varepsilon \rightarrow 0} \int_{0}^{T}\left\|\nabla u^{\varepsilon}(t, x)-\frac{1}{\varepsilon} \nabla_{y} u_{0} \star \omega_{\delta}\left(\frac{t}{\varepsilon}, x, \frac{x}{\varepsilon}\right)\right\|_{2}^{2}=0,
\end{aligned}
$$

where $\left(p_{0}, u_{0}\right) \in C\left([0, T) ; L^{2}\left(\mathbb{R}^{d}\right) \times L^{2}\left(\mathbb{R}^{d}\right)^{d}\right)$ is the unique solution of (3.4). If in addition $\left(p_{0}, u_{0}\right)$ are admissible test functions for each $t \in[0, T]$, then (3.3) still holds without the necessity of using a mollification.

\section{Proof of the main results}

The existence of a solution $\left(p^{\varepsilon}, u^{\varepsilon}\right) \in C\left([0, T) ; L^{2}\left(\mathbb{R}_{\varepsilon}^{d}\right) \times L^{2}\left(\mathbb{R}_{\varepsilon}^{d}\right)^{d}\right)$ to the linear system (1.1) is very classical and we do not recall it here. We only observe that the solution $\left(p^{\varepsilon}, u^{\varepsilon}\right)$ satisfies

$$
\left\|p^{\varepsilon}(t)\right\|_{L^{2}\left(\mathbb{R}_{\varepsilon}^{d}\right)}^{2}+\left\|u^{\varepsilon}(t)\right\|_{L^{2}\left(\mathbb{R}_{\varepsilon}^{d}\right)}^{2}+2 \varepsilon^{\beta} \int_{0}^{t}\left\|\nabla u^{\varepsilon}\right\|_{L^{2}\left(\mathbb{R}_{\varepsilon}^{d}\right)}^{2}=\left\|b^{\varepsilon}\right\|_{L^{2}\left(\mathbb{R}_{\varepsilon}^{d}\right)}^{2}+\left\|a^{\varepsilon}\right\|_{L^{2}\left(\mathbb{R}_{\varepsilon}^{d}\right)}^{2} .
$$

We note that the proofs of the main results repeatedly make use of the Poincaré inequality and the energy estimate satisfied by the sequence of solutions $\left(p^{\varepsilon}, u^{\varepsilon}\right)$. We state the Poincaré inequality on the domain $\mathbb{R}_{\varepsilon}^{d}$ (see [18]):

Theorem 4.1. Let $f \in H_{0}^{1}\left(\mathbb{R}_{\varepsilon}^{d}\right)$. Then we have

$$
\|f\|_{L^{2}\left(\mathbb{R}_{\varepsilon}^{d}\right)} \leq C \varepsilon\|\nabla f\|_{L^{2}\left(\mathbb{R}_{\varepsilon}^{d}\right)} .
$$


4.1. $(\beta<0)$ Proof of Theorem 3.1.

Proof. Let $u_{0}$ be the solution of the limit system (2.7) and define the following error terms:

$$
\begin{aligned}
\alpha^{\varepsilon} & =p^{\varepsilon}-b_{0}\left(x, \frac{x}{\varepsilon}\right), \\
\beta^{\varepsilon} & =u^{\varepsilon}-u_{0}\left(\frac{t}{\varepsilon^{2-\beta}}, x, \frac{x}{\varepsilon}\right) .
\end{aligned}
$$

Hence, we see that $\left(\alpha^{\varepsilon}, \beta^{\varepsilon}\right)$ satisfies

$$
\left\{\begin{array}{rlrl}
\partial_{t} \alpha^{\varepsilon}+\operatorname{div} \beta^{\varepsilon} & =F^{\varepsilon}(t, x) & & \text { in }(0, \infty) \times \mathbb{R}_{\varepsilon}^{d}, \\
\partial_{t} \beta^{\varepsilon}-\varepsilon^{\beta} \triangle \beta^{\varepsilon}+\nabla \alpha^{\varepsilon} & =G^{\varepsilon}(t, x), & & \text { on } \partial \mathbb{R}_{\varepsilon}^{d}, \\
\beta^{\varepsilon} & =0 & & \\
\beta^{\varepsilon}(t=0) & =a^{\varepsilon}(x)-a_{0}\left(x, \frac{x}{\varepsilon}\right), \\
\alpha^{\varepsilon}(t=0) & =b^{\varepsilon}(x)-b_{0}\left(x, \frac{x}{\varepsilon}\right), & &
\end{array}\right.
$$

where the force terms are given by

$$
\begin{aligned}
& F^{\varepsilon}(t, x)=\left(-\operatorname{div}_{x} u_{0}-\varepsilon^{-1} \operatorname{div}_{y} u_{0}\right)\left(t, x, \frac{x}{\varepsilon}\right) \\
& G^{\varepsilon}(t, x)=\left(\varepsilon^{\beta} \triangle_{x} u_{0}+2 \varepsilon^{\beta-1} \operatorname{div}_{y} \nabla_{x} u_{0}-\nabla b_{0}\right)\left(t, x, \frac{x}{\varepsilon}\right) .
\end{aligned}
$$

Here $\nabla=\nabla_{x}+\frac{1}{\varepsilon} \nabla_{y}$.

Remark 4.2. Due to the parabolic regularizing effect of the heat Equation (2.7), the trace of the terms $\partial_{x}^{\alpha} \partial_{y}^{\gamma} u_{0}\left(\frac{t}{\varepsilon^{2-\beta}}, x, \frac{x}{\varepsilon}\right)$ are well defined for $|\alpha| \leq 2$. We refer the reader to [2] for details.

The energy equality for (4.1) is given by

$$
\begin{aligned}
& \left\|\alpha^{\varepsilon}(t)\right\|_{L^{2}\left(\mathbb{R}_{\varepsilon}^{d}\right)}^{2}+\left\|\beta^{\varepsilon}(t)\right\|_{L^{2}\left(\mathbb{R}_{\varepsilon}^{d}\right)}^{2}+\varepsilon^{\beta} \int_{0}^{t}\left\|\nabla \beta^{\varepsilon}(s)\right\|_{L^{2}\left(\mathbb{R}_{\varepsilon}^{d}\right)}^{2} \\
= & \left\|\alpha^{\varepsilon}(0)\right\|_{L^{2}\left(\mathbb{R}_{\varepsilon}^{d}\right)}^{2}+\left\|\beta^{\varepsilon}(0)\right\|_{L^{2}\left(\mathbb{R}_{\varepsilon}^{d}\right)}^{2}+\left(\int_{0}^{t} \int_{\mathbb{R}_{\varepsilon}^{d}}\left(F^{\varepsilon} \alpha^{\varepsilon}+G^{\varepsilon} \cdot \beta^{\varepsilon}\right)\right),
\end{aligned}
$$

and we now estimate the term

$$
\left(\int_{0}^{t} \int_{\mathbb{R}_{\varepsilon}^{d}}\left(F^{\varepsilon} \alpha^{\varepsilon}+G^{\varepsilon} \cdot \beta^{\varepsilon}\right)\right) .
$$

We write

$$
\int_{0}^{t} \int_{\mathbb{R}_{\varepsilon}^{d}} F^{\varepsilon} \alpha^{\varepsilon}=F_{1}+F_{2},
$$


and we obtain the following bounds:

$$
\begin{aligned}
\left|F_{1}\right| & \leq C \int_{0}^{t}\left\|\operatorname{div}_{x} u_{0}\left(\frac{s}{\varepsilon^{2-\beta}}, x, \frac{x}{\varepsilon}\right)\right\|_{L^{2}\left(\mathbb{R}_{\varepsilon}^{d}\right)}^{2} d s+\int_{0}^{t}\left\|\alpha^{\varepsilon}(s)\right\|_{L^{2}\left(\mathbb{R}_{\varepsilon}^{d}\right)}^{2} \\
& \leq C \varepsilon^{2-\beta} \int_{0}^{\infty}\left\|\operatorname{div}_{x} u_{0}\left(\tau, x, \frac{x}{\varepsilon}\right)\right\|_{L^{2}\left(\mathbb{R}_{\varepsilon}^{d}\right)}^{2} d \tau+\int_{0}^{t}\left\|\alpha^{\varepsilon}(s)\right\|_{L^{2}\left(\mathbb{R}_{\varepsilon}^{d}\right)}^{2} \\
& \leq C \varepsilon^{2-\beta} \int_{0}^{\infty}\left\|\operatorname{div}_{x} u_{0}\left(\tau, x, \frac{x}{\varepsilon}\right)\right\|_{L^{2}\left(\mathbb{R}^{d} \times Y_{f}\right)}^{2} d \tau+\int_{0}^{t}\left\|\alpha^{\varepsilon}(s)\right\|_{L^{2}\left(\mathbb{R}_{\varepsilon}^{d}\right)}^{2} \\
& \leq C \varepsilon^{2-\beta}+\int_{0}^{t}\left\|\alpha^{\varepsilon}(s)\right\|_{L^{2}\left(\mathbb{R}_{\varepsilon}^{d}\right)}^{2}, \\
\left|F_{2}\right| & \leq \frac{C}{\varepsilon^{2}} \int_{0}^{t}\left\|\operatorname{div}_{y} u_{0}\left(\frac{s}{\varepsilon^{2-\beta}}, x, \frac{x}{\varepsilon}\right)\right\|_{L^{2}\left(\mathbb{R}_{\varepsilon}^{d}\right)}^{2}+\int_{0}^{t}\left\|\alpha^{\varepsilon}(s)\right\|_{L^{2}\left(\mathbb{R}_{\varepsilon}^{d}\right)}^{2} \\
& \leq \frac{C}{\varepsilon^{2}} \varepsilon^{2-\beta} \int_{0}^{\infty}\left\|\operatorname{div}_{y} u_{0}\left(\tau, x, \frac{x}{\varepsilon}\right)\right\|_{L^{2}\left(\mathbb{R}_{\varepsilon}^{d}\right)}^{2} d \tau+\int_{0}^{t}\left\|\alpha^{\varepsilon}(s)\right\|_{L^{2}\left(\mathbb{R}_{\varepsilon}^{d}\right)}^{2} \\
& \leq C \varepsilon^{-\beta}+\int_{0}^{t}\left\|\alpha^{\varepsilon}(s)\right\|_{L^{2}\left(\mathbb{R}_{\varepsilon}^{d}\right)}^{2} .
\end{aligned}
$$

We now explain the estimates above. The first inequality in the estimates of $F_{1}, F_{2}$ is just an application of the Cauchy-Schwartz inequality, while the second inequality is just the change of variable $s=\varepsilon^{2-\beta} \tau$. The third inequality in $F_{1}, F_{2}$ follows from strong two-scale convergence (see (2.4)) and hence holds for $\varepsilon$ small enough.

We now justify the bound on $\operatorname{div}_{x} u_{0}(\tau, x, y)$ and $\operatorname{div}_{y} u_{0}$. First, notice that $\partial_{x}^{\alpha} u_{0}$ satisfies the same system $(2.7)$ as $u_{0}$ with the initial data $\partial_{x}^{\alpha} u_{0}(t=0)=\partial_{x}^{\alpha} a_{0}$. The energy equality reads

$$
\left\|\partial_{x}^{\alpha} u_{0}(\tau)\right\|_{L^{2}\left(\mathbb{R}^{d} \times Y_{f}\right)}^{2}+2 \int_{0}^{\tau}\left\|\partial_{x}^{\alpha} \nabla_{y} u_{0}\left(\tau^{\prime}\right)\right\|_{L^{2}\left(\mathbb{R}^{d} \times Y_{f}\right)}^{2}=\left\|\partial_{x}^{\alpha} a_{0}\right\|_{L^{2}\left(\mathbb{R}^{d} \times Y_{f}\right)}^{2} .
$$

We take $\alpha=0$, and deduce that

$$
\int_{0}^{\infty}\left\|\operatorname{div}_{y} u_{0}(\tau, x, y)\right\|_{L^{2}\left(\mathbb{R}^{d} \times Y_{f}\right)}^{2} d \tau \leq C .
$$

On the other hand, using the Poincaré inequality in the cell $Y_{f}$, we also deduce that

$$
\int_{0}^{\tau}\left\|\partial_{x}^{\alpha} u_{0}\left(\tau^{\prime}\right)\right\|_{L^{2}\left(\mathbb{R}^{d} \times Y_{f}\right)}^{2} \leq C \int_{0}^{\tau}\left\|\partial_{x}^{\alpha} \nabla_{y} u_{0}\left(\tau^{\prime}\right)\right\|_{L^{2}\left(\mathbb{R}^{d} \times Y_{f}\right)}^{2}
$$

for all $|\alpha| \leq 2$. Hence,

$$
\int_{0}^{\infty}\left\|\operatorname{div}_{x} u_{0}(\tau, x, y)\right\|_{L^{2}\left(\mathbb{R}^{d} \times Y_{f}\right)}^{2} \leq C .
$$

Similarly, we write

$$
\int_{0}^{t} \int_{\mathbb{R}_{\varepsilon}^{d}} G^{\varepsilon} \cdot \beta^{\varepsilon}=G_{1}+G_{2}+G_{3}
$$


and we obtain the bounds

$$
\begin{aligned}
\left|G_{1}\right| & \leq C \varepsilon^{\beta}\left(\int_{0}^{t}\left\|\triangle_{x} u_{0}\left(\frac{s}{\varepsilon^{2-\beta}}, x, \frac{x}{\varepsilon}\right)\right\|_{L^{2}\left(\mathbb{R}_{\varepsilon}^{d}\right)}^{2}\right)^{1 / 2}\left(\int_{0}^{t}\left\|\beta^{\varepsilon}(s)\right\|_{L^{2}\left(\mathbb{R}_{\varepsilon}^{d}\right)}^{2}\right)^{1 / 2} \\
& \leq C \varepsilon^{\beta+1}\left(\int_{0}^{t}\left\|\triangle_{x} u_{0}\left(\frac{s}{\varepsilon^{2-\beta}}, x, \frac{x}{\varepsilon}\right)\right\|_{L^{2}\left(\mathbb{R}_{\varepsilon}^{d}\right)}^{2}\right)^{1 / 2}\left(\int_{0}^{t}\left\|\nabla \beta^{\varepsilon}(s)\right\|_{L^{2}\left(\mathbb{R}_{\varepsilon}^{d}\right)}^{2}\right)^{1 / 2} \\
& \leq C \varepsilon^{2+\beta} \int_{0}^{t}\left\|\triangle_{x} u_{0}\left(\frac{s}{\varepsilon^{2-\beta}}, x, \frac{x}{\varepsilon}\right)\right\|_{L^{2}\left(\mathbb{R}_{\varepsilon}^{d}\right)}^{2}+\frac{\varepsilon^{\beta}}{4} \int_{0}^{t}\left\|\nabla \beta^{\varepsilon}(s)\right\|_{L^{2}\left(\mathbb{R}_{\varepsilon}^{d}\right)}^{2} \\
& \leq C \varepsilon^{4}+\frac{\varepsilon^{\beta}}{4} \int_{0}^{t}\left\|\nabla \beta^{\varepsilon}(s)\right\|_{L^{2}\left(\mathbb{R}_{\varepsilon}^{d}\right)}^{2}, \\
\left|G_{2}\right| \leq & C \varepsilon^{\beta-1}\left(\int_{0}^{t}\left\|\operatorname{div} \nabla_{x} u_{0}\left(\frac{s}{\varepsilon^{2-\beta}}, x, \frac{x}{\varepsilon}\right)\right\|_{L^{2}\left(\mathbb{R}_{\varepsilon}^{d}\right)}^{2}\right)^{1 / 2}\left(\int_{0}^{t}\left\|\beta^{\varepsilon}(s)\right\|_{L^{2}\left(\mathbb{R}_{\varepsilon}^{d}\right)}\right)^{1 / 2} \\
\leq & C \varepsilon^{\beta}\left(\int_{0}^{t}\left\|\operatorname{div}_{y} \nabla_{x} u_{0}\left(\frac{s}{\varepsilon^{2-\beta}}, x, \frac{x}{\varepsilon}\right)\right\|_{L^{2}\left(\mathbb{R}_{\varepsilon}^{d}\right)}^{2}\right)^{1 / 2}\left(\int_{0}^{t}\left\|\nabla \beta^{\varepsilon}(s)\right\|_{L^{2}\left(\mathbb{R}_{\varepsilon}^{d}\right)}\right)^{1 / 2} \\
\leq & C \varepsilon^{\beta} \int_{0}^{t}\left\|\operatorname{div}_{y} \nabla_{x} u_{0}\left(\frac{s}{\varepsilon^{2-\beta}}, x, \frac{x}{\varepsilon}\right)\right\|_{L^{2}\left(\mathbb{R}_{\varepsilon}^{d}\right)}^{2}+\frac{\varepsilon^{\beta}}{4} \int_{0}^{t}\left\|\nabla \beta^{\varepsilon}(s)\right\|_{L^{2}\left(\mathbb{R}_{\varepsilon}^{d}\right)}^{2} \\
\leq & C \varepsilon^{2}+\frac{\varepsilon^{\beta}}{4} \int_{0}^{t}\left\|\nabla \beta^{\varepsilon}(s)\right\|_{L^{2}\left(\mathbb{R}_{\varepsilon}^{d}\right)}^{2}, \\
\leq & C t \varepsilon^{-\beta}+\frac{\varepsilon^{\beta}}{4} \int_{0}^{t}\left\|\nabla \beta^{\varepsilon}(s)\right\|_{L^{2}\left(\mathbb{R}_{\varepsilon}^{d}\right)}^{2} . \\
\leq & \left|\int_{0}^{t} \int_{\mathbb{R}_{\varepsilon}^{d}} b_{0}\left(x, \frac{x}{\varepsilon}\right) \operatorname{div} \beta^{\varepsilon}(s, x)\right| \\
\leq & \left(\int_{0}^{t}\left\|b_{0}\left(x, \frac{x}{\varepsilon}\right)\right\|_{L^{2}\left(\mathbb{R}_{\varepsilon}^{d}\right)}^{2} \int^{t}\left\|b_{0}\left(x, \frac{x}{\varepsilon}\right)\right\|_{L^{2}\left(\mathbb{R}_{\varepsilon}^{d}\right)}^{2}+\frac{\varepsilon^{\beta}}{4} \int_{0}^{t}\left\|\nabla \beta^{\varepsilon}(s)\right\|_{L^{2}\left(\mathbb{R}_{\varepsilon}^{d}\right)}^{t}\left\|\nabla \beta^{\varepsilon}(s)\right\|_{L^{2}\left(\mathbb{R}_{\varepsilon}^{d}\right)}^{2}\right)^{1 / 2} \\
\leq &
\end{aligned}
$$

Here, the second inequality in the estimates of $G_{1}, G_{2}$ made use of the Poincaré inequality on the domain $\mathbb{R}_{\varepsilon}^{d}$. We concluded the final inequality in the estimates $G_{1}, G_{2}$ with a change of variable in time along with the bounds in (4.4), (4.5).

Combining all the estimates on the force terms, the energy bound (4.2) becomes

$$
\begin{gathered}
\left\|\alpha^{\varepsilon}(t)\right\|_{L^{2}\left(\mathbb{R}_{\varepsilon}^{d}\right)}^{2}+\left\|\beta^{\varepsilon}(t)\right\|_{L^{2}\left(\mathbb{R}_{\varepsilon}^{d}\right)}^{2}+\frac{\varepsilon^{\beta}}{4} \int_{0}^{t}\left\|\nabla \beta^{\varepsilon}(s)\right\|_{L^{2}\left(\mathbb{R}_{\varepsilon}^{d}\right)}^{2} \\
\leq\left\|\alpha^{\varepsilon}(0)\right\|_{L^{2}\left(\mathbb{R}_{\varepsilon}^{d}\right)}^{2}+\left\|\beta^{\varepsilon}(0)\right\|_{L^{2}\left(\mathbb{R}_{\varepsilon}^{d}\right)}^{2}+\int_{0}^{t}\left\|\alpha^{\varepsilon}(s)\right\|_{L^{2}\left(\mathbb{R}_{\varepsilon}^{d}\right)}^{2}+C_{t, \varepsilon},
\end{gathered}
$$

where

$$
C_{t, \varepsilon}=C\left(\varepsilon^{2-\beta}+\varepsilon^{-\beta}+\varepsilon^{4}+\varepsilon^{2}+t \varepsilon^{-\beta}\right) .
$$

The proof of the Theorem 3.1 now follows by applying Gronwall's lemma to (4.6). 


\subsection{Proof of Theorem 3.2.}

Proof. In this Theorem, $x$ is just a parameter. Hence, without loss of generality, we can assume that $b_{0}$ only depends on $y$. We begin with the proof of (i), the existence and uniqueness for the limit system (2.7). We rewrite the vector equation of system (2.7) as

$$
u_{1}=\triangle_{D}^{-1} \nabla p_{0},
$$

where $\triangle_{D}^{-1}$ is the inverse Laplacian in the $y$ variable with Dirichlet boundary condition on $\partial Y_{s}$ and periodic boundary conditions on $\partial Y$. Hence, the scalar equation of (2.7) becomes

$$
\left\{\begin{aligned}
\partial_{t} p_{0} & =-\operatorname{div} \triangle_{D}^{-1} \nabla p_{0} \quad \text { in }(0, \infty) \times Y_{f}, \\
p_{0}(t=0) & =b_{0} .
\end{aligned}\right.
$$

We can also apply $\partial_{t}$ to (4.7) and use the scalar equation of system (2.7) to obtain the initial value problem for $u_{1}$ :

$$
\left\{\begin{aligned}
\partial_{t} u_{1} & =-\triangle_{D}^{-1} \nabla \operatorname{div} u_{1} \quad \text { in }(0, \infty) \times Y_{f}, \\
u_{1}(t=0) & =\triangle_{D}^{-1} \nabla b_{0} .
\end{aligned}\right.
$$

The proof of (i) now follows as a result of the explicit solutions for $p_{0}$ and $u_{1}$,

$$
p_{0}=e^{-t S} b_{0}, \quad u_{1}=e^{-t L} u_{1}(t=0),
$$

where $S=\operatorname{div} \triangle_{D}^{-1} \nabla$ and $L=\triangle_{D}^{-1} \nabla \operatorname{div}$.

Proposition 4.3. The operator $S$ is a positive, self-adjoint, continuous mapping on $L^{2}\left(Y_{f}\right)$ and the operator $L$ is a positive, self-adjoint, continuous mapping on $H_{0 \#}^{1}\left(Y_{f}\right)^{d}$. The operators $S$ and $L$ are related by the following property: for all $u \in H_{0 \#}^{1}\left(Y_{f}\right)^{d}$ and $p \in L^{2}\left(Y_{f}\right)$ we have

$$
\text { divLu }=\text { Sdivu }, \quad L \triangle_{D}^{-1} \nabla p=\triangle_{D}^{-1} \nabla S p .
$$

Moreover, there exists a constant $\lambda$ such that for $p \in L_{0}^{2}\left(Y_{f}\right)$, we have

$$
\|p\|_{L^{2}\left(Y_{f}\right)} \leq \lambda\|S p\|_{L^{2}\left(Y_{f}\right)}
$$

Proof. By elliptic regularity, we deduce that $S$ is bounded on $L^{2}\left(Y_{f}\right)$. Taking the inner product

$$
\begin{aligned}
(S p, p)_{L^{2}\left(Y_{f}\right)} & =\left(\operatorname{div} \triangle_{D}^{-1} \nabla p, p\right)_{L^{2}\left(Y_{f}\right)}=-\left\langle\nabla p, \triangle_{D}^{-1} \nabla p\right\rangle \\
& =-\langle\Delta u, u\rangle=\left\|\nabla \triangle_{D}^{-1} \nabla p\right\|_{L^{2}\left(Y_{f}\right)}^{2}
\end{aligned}
$$

where $u=\triangle_{D}^{-1} \nabla p$ and

$$
\langle\cdot, \cdot\rangle=\langle\cdot, \cdot\rangle_{H^{-1} \times H_{0}^{1}}
$$

denotes the duality bracket between the spaces $H^{-1}\left(Y_{f}\right)$ and $H_{0}^{1}\left(Y_{f}\right)$. By a similar argument, we deduce that

$$
(L u, v)_{H_{0 \#}^{1}\left(Y_{f}\right)}=(\operatorname{div} u, \operatorname{div} v)_{L^{2}\left(Y_{f}\right)} .
$$


To prove (4.11), we present two different methods. The first one uses the following estimate of the stationary Stokes system

$$
\begin{aligned}
& \left\{\begin{aligned}
-\Delta u+\nabla p=f & \text { in } Y_{f}, \\
\operatorname{div}(u)=g & \text { in } Y_{f}, \\
u=0 & \text { on } \partial Y_{f}, \\
\int_{Y_{f}} p=0, &
\end{aligned}\right. \\
& \|p\|_{L^{2}}+\|\nabla u\|_{L^{2}} \leq C\left[\|g\|_{L^{2}}+\|f\|_{H^{-1}}\right] .
\end{aligned}
$$

We use (4.14) with $f=0$ and $g=\operatorname{div}(u)=\operatorname{div} \triangle_{D}^{-1} \nabla p=S p$. Hence, we deduce that

$$
\|p\|_{L^{2}}+\|\nabla u\|_{L^{2}} \leq C\|S p\|_{L^{2}} .
$$

The second proof uses the so-called Bogovskii operator $\mathcal{R}$. Indeed, for $1<p<\infty$, there exists a linear operator $\mathcal{R}$ (which is not unique) such that

$$
\mathcal{R}: L_{0}^{p}\left(Y_{f}\right)=\left\{f \in L^{p}\left(Y_{f}\right) \mid \int_{Y_{f}} f=0\right\} \rightarrow W_{0}^{1, p}\left(Y_{f}\right)
$$

and $v=\mathcal{R} f$ solves the equation

$$
\operatorname{div}(v)=f \quad \text { in } Y_{f}, \quad v=0 \quad \text { on } \partial Y_{f} .
$$

The existence of such a $\mathcal{R}$ that maps $L_{0}^{p}\left(\Omega_{\varepsilon}\right)$ into $W_{0}^{1, p}$ is well known (see for instance $[4,10])$. We will only use $\mathcal{R}$ with $p=2$. It allows us to prove that for $p \in L_{0}^{2}\left(Y_{f}\right)$, we have $\|p\|_{L_{0}^{2}}\left(Y_{f}\right) \leq C\|\nabla p\|_{H^{-1}}$. Indeed, we have

$$
\begin{aligned}
\|p\|_{L_{0}^{2}\left(Y_{f}\right)} & =\sup _{\|g\|_{L_{0}^{2}\left(Y_{f}\right)}=1} \int_{Y_{f}} p g=\sup _{\|g\|_{L_{0}^{2}\left(Y_{f}\right)}=1} \int_{Y_{f}} p \operatorname{div}(\mathcal{R} g) \\
& \leq \sup _{\|g\|_{L_{0}^{2}\left(Y_{f}\right)}=1}\|\nabla p\|_{H^{-1}}\|\mathcal{R} g\|_{H_{0}^{1}\left(Y_{f}\right)} \\
& \leq C\|\nabla p\|_{H^{-1}} .
\end{aligned}
$$

Hence, we deduce that $\|p\|_{L_{0}^{2}} \leq C\left\|\triangle_{D}^{-1} \nabla p\right\|_{H_{0}^{1}} \leq C\|S p\|_{L^{2}}$.

We now proceed with the proof of (ii). Notice that repeatedly taking time derivatives of the scalar equation in (2.7) and the vector system (4.9) yields the estimates

$$
\begin{gathered}
\left\|\partial_{t}^{k} p_{0}(t)\right\|_{L^{2}\left(Y_{f}\right)}=\left\|\partial_{t}^{k-1} \operatorname{div} u_{1}(t)\right\|_{L^{2}\left(Y_{f}\right)}, \\
\left\|\partial_{t}^{k} u_{1}(t)\right\|_{H_{0 \#}^{1}\left(Y_{f}\right)}=\left\|\partial_{t}^{k-1} \nabla \operatorname{div} u_{1}(t)\right\|_{H^{-1}\left(Y_{f}\right)} \leq\left\|\partial_{t}^{k-1} \operatorname{div} u_{1}(t)\right\|_{L^{2}\left(Y_{f}\right)},
\end{gathered}
$$

where $k=1,2, \ldots$. The proof of (ii) now follows by the energy bound for $\left(p_{0}, u_{1}\right)$

$$
\left\|p_{0}(t)\right\|_{L^{2}\left(Y_{f}\right)}^{2}+\int_{0}^{t}\left\|\nabla u_{1}(s)\right\|_{L^{2}\left(Y_{f}\right)}^{2}=\left\|b_{0}\right\|_{L^{2}\left(Y_{f}\right)}^{2} .
$$


For the proof of (iii) we note that it suffices to prove the bounds associated with the pressure, since the bounds for $u_{1}$ are obtained from the vector equation in (2.7) and standard elliptic regularity estimates. We define

$$
q_{0}=p_{0}-f_{Y_{f}} b_{0}
$$

Observe that $\left(q_{0}, u_{1}\right)$ solves the system $(2.7)$ with initial value $q_{0}(t=0)=b_{0}-f_{Y_{f}} b_{0}$. Moreover, the solution $\left(q_{0}, u_{1}\right)$ satisfies

$$
\frac{d}{d t}\left\|q_{0}(t)\right\|_{L^{2}\left(Y_{f}\right)}^{2}+2\left(S q_{0}(t), q_{0}(t)\right)_{L^{2}\left(Y_{f}\right)}=0
$$

and as a result we get, from the coercivity estimate (4.11),

$$
\partial_{t}\left\|q_{0}(t)\right\|_{L^{2}\left(Y_{f}\right)}^{2}+C\left\|q_{0}(t)\right\|_{L^{2}\left(Y_{f}\right)}^{2} \leq 0
$$

Applying Gronwall's lemma to (4.19) gives us exponential decay:

$$
\|q(t)\|_{L^{2}\left(Y_{f}\right)}^{2} \leq e^{-C t}\|q(0)\|_{L^{2}\left(Y_{f}\right)}^{2} .
$$

Thus the proof of (iii) is complete. The proof of Theorem 3.2 is now complete.

REMARK 4.4. We end this subsection by some few extra remarks about the operators $S$ and $L$. We think these properties are interesting by themselves but will not be used in the rest of the paper. First, it is clear that $S$ and $L$ have the same eigenvalues. Indeed, 0 is an eigenvalue for both of them and $\operatorname{Ker}(S)=\{p \in$ $L^{2}\left(Y_{f}\right) \mid p$ is constant $\}, \operatorname{Ker}(L)=\left\{u \in H_{0 \#}^{1}\left(Y_{f}\right) \mid \operatorname{div}(u)=0\right\}$. Moreover, if $(p, \lambda)$ satisfies $S p=\lambda p$ and $\lambda \neq 0$ then, by the second equation in (4.10), we deduce that $L \triangle_{D}^{-1} \nabla p=\triangle_{D}^{-1} \nabla S p=\lambda \triangle_{D}^{-1} \nabla p$ and if $(u, \lambda)$ satisfies $L u=\lambda u$ and $\lambda \neq 0$, then by the first equation in (4.10), we deduce that $S \operatorname{div} u=\operatorname{div} L u=\lambda \operatorname{div} u$.

Another important property is the fact that if $(p, \lambda)$ satisfies $S p=\lambda p$, namely $\operatorname{div} \triangle_{D}^{-1} \nabla p=\lambda p$, then taking the Laplacian and using that it commutes with div we deduce that $\operatorname{div} \nabla p=\Delta p=\lambda \Delta p$. Hence, we deduce that either $\lambda=1$ or $\Delta p=0$. Moreover, if we define $H=\left\{p \in L^{2}\left(Y_{f}\right) \mid \Delta p=0\right\}$ and denote by $H^{\perp}$ its orthogonal complement in $L^{2}\left(Y_{f}\right)$, namely $L^{2}\left(Y_{f}\right)=H \oplus H^{\perp}$, hence $S_{H^{\perp}}=I d$.

We can also show that if $p_{0}$ solves (4.8) and that $\partial_{y}^{\gamma} \Delta b_{0} \in L^{q}\left(Y_{f}\right)$ for some $q \geq 1$ and $\gamma \in \mathbb{N}^{d}$, then we get the exponential decay of $\partial_{y}^{\gamma} \Delta p_{0}$ in $L^{q}\left(Y_{f}\right)$. Indeed, applying the derivative $\triangle \partial_{y}^{\gamma}$ to the scalar equation in (2.7) yields that

$$
\partial_{t} \triangle \partial_{y}^{\gamma} p_{0}+\triangle \partial_{y}^{\gamma} p_{0}=0
$$

holds in the sense of distributions, and hence $\partial_{y}^{\gamma} \Delta p_{0}=e^{-t} \partial_{y}^{\gamma} \Delta b_{0}$. However, this does not allow to show that if $b_{0} \in H^{s}\left(Y_{f}\right)$, then $p_{0}$ decays in $H^{s}\left(Y_{f}\right)$. All we can say is that for each $s \in \mathbb{N}$, there exists a constant $C_{s}$ such that for $b_{0} \in H^{s}\left(Y_{f}\right)$, the solution $p_{0}$ of (4.8) satisfies the bound $\left\|p_{0}(t)\right\|_{H^{s}\left(Y_{f}\right)} \leq e^{C_{s} t}\left\|b_{0}(t)\right\|_{H^{s}\left(Y_{f}\right)}$. Indeed, we have just to observe that, using elliptic regularity, we can deduce that the restriction of the operator $S$ to $H^{s}\left(Y_{f}\right)$ defines a bounded operator from $H^{s}\left(Y_{f}\right)$ to $H^{s}\left(Y_{f}\right)$. 
4.3. $(0<\beta<1)$ Proof of Theorem 3.3.

Proof. We define the following error terms:

$$
\begin{aligned}
& \alpha^{\varepsilon}=p^{\varepsilon}-p_{0}\left(\frac{t}{\varepsilon^{\beta}}, x, \frac{x}{\varepsilon}\right)-\varepsilon^{(1-\beta)} p_{1}\left(\frac{t}{\varepsilon^{2-\beta}}, x, \frac{x}{\varepsilon}\right), \\
& \beta^{\varepsilon}=u^{\varepsilon}-u_{0}\left(\frac{t}{\varepsilon^{2-\beta}}, x, \frac{x}{\varepsilon}\right)-\varepsilon^{(1-\beta)} u_{1}\left(\frac{t}{\varepsilon^{\beta}}, x, \frac{x}{\varepsilon}\right),
\end{aligned}
$$

where $\left(p_{0}, u_{1}\right)$ satisfy $(2.7)$ and $u_{0}$ is obtained from the heat Equation (2.8). The remaining pressure term, $p_{1}$, is defined by

$$
p_{1}(\tau, x, y)=\int_{\tau}^{\infty} \operatorname{div}_{y} u_{0}\left(\tau^{\prime}, x, y\right) d \tau^{\prime}
$$

The error term $\left(\alpha^{\varepsilon}, \beta^{\varepsilon}\right)$ defined in (4.20) satisfies the system given in (4.1), but with force terms equal to

$$
\begin{aligned}
F^{\varepsilon}(t, x)=- & \operatorname{div}_{x} u_{0}-\varepsilon^{1-\beta} \operatorname{div}_{x} u_{1} \\
G^{\varepsilon}(t, x)=- & \varepsilon^{1-2 \beta} \partial_{\rho} u_{1}+\varepsilon^{\beta} \triangle_{x} u_{0}+2 \varepsilon^{\beta-1} \operatorname{div}_{y} \nabla_{x} u_{0}+\varepsilon \triangle_{x} u_{1}, \\
& +2 \operatorname{div}_{y} \nabla_{x} u_{1}-\nabla_{x} p_{0}-\varepsilon^{1-\beta} \nabla p_{1},
\end{aligned}
$$

where $\left(p_{0}, u_{1}\right)$ are functions of $(\rho, x, y)$ and $\left(u_{0}, p_{1}\right)$ are functions of $(\tau, x, y)$ and $\rho=$ $t / \varepsilon^{\beta}, \tau=t / \varepsilon^{2-\beta}$ and $y=x / \varepsilon$.

We now imitate the proof given for the case $\beta<0$. Notice that $\left(\alpha^{\varepsilon}, \beta^{\varepsilon}\right)$ satisfies the energy bound in (4.2), hence it suffices to show

$$
\begin{aligned}
& \left|\int_{0}^{t} \int_{\mathbb{R}_{\varepsilon}^{d}} F^{\varepsilon} \alpha^{\varepsilon}+G^{\varepsilon} \cdot \beta^{\varepsilon}\right| \\
\leq & O\left(\varepsilon^{\gamma}\right)+C_{1} \varepsilon^{\beta} \int_{0}^{t}\left\|\nabla \beta^{\varepsilon}(s)\right\|_{L^{2}\left(\mathbb{R}_{\varepsilon}^{d}\right)}^{2}+C \int_{0}^{t}\left\|\alpha^{\varepsilon}(s)\right\|_{L^{2}\left(\mathbb{R}_{\varepsilon}^{d}\right)}^{2}
\end{aligned}
$$

for some $0<\gamma$ and constant $C_{1}<1$.

We begin by writing

$$
\int_{0}^{t} \int_{\mathbb{R}_{\varepsilon}^{d}} F^{\varepsilon} \alpha^{\varepsilon}=F_{1}+F_{2}
$$

and we obtain the following inequalities:

$$
\begin{aligned}
\left|F_{1}\right| & \leq C \int_{0}^{t}\left\|\operatorname{div}_{x} u_{0}\left(\frac{s}{\varepsilon^{2-\beta}}, x, \frac{x}{\varepsilon}\right)\right\|_{L^{2}\left(\mathbb{R}_{\varepsilon}^{d}\right)}^{2}+\int_{0}^{t}\left\|\alpha^{\varepsilon}(s)\right\|_{L^{2}\left(\mathbb{R}_{\varepsilon}^{d}\right)}^{2} \\
& \leq C \varepsilon^{2-\beta}+\int_{0}^{t}\left\|\alpha^{\varepsilon}(s)\right\|_{L^{2}\left(\mathbb{R}_{\varepsilon}^{d}\right)}^{2}, \\
\left|F_{2}\right| & \leq C \varepsilon^{2-2 \beta}\left\|\operatorname{div}_{x} u_{1}\left(\frac{s}{\varepsilon^{\beta}}, x, \frac{x}{\varepsilon}\right)\right\|_{L^{2}\left(\mathbb{R}_{\varepsilon}^{d}\right)}^{2}+\int_{0}^{t}\left\|\alpha^{\varepsilon}(s)\right\|_{L^{2}\left(\mathbb{R}_{\varepsilon}^{d}\right)}^{2} \\
& \leq C \varepsilon^{2-\beta}+\int_{0}^{t}\left\|\alpha^{\varepsilon}(s)\right\|_{L^{2}\left(\mathbb{R}_{\varepsilon}^{d}\right)}^{2} .
\end{aligned}
$$

Note that the estimate associated to the term $F_{2}$ made use of the energy bound (4.18), as well as the Poincaré inequality

$$
\left\|\partial_{x}^{\alpha} u_{1}(t)\right\|_{L^{2}\left(Y_{f}\right)} \leq C\left\|\partial_{x}^{\alpha} \nabla u_{1}(t)\right\|_{L^{2}\left(Y_{f}\right)} .
$$


Similarly we write

$$
\int_{0}^{t} \int_{\mathbb{R}_{\varepsilon}^{d}} G^{\varepsilon} \cdot \beta^{\varepsilon}=\sum_{j=1}^{7} G_{j}
$$

and we proceed to bound the terms $G_{j}, j=1,2, \ldots 6$, followed by estimates for the term $G_{7}$. We have

$$
\begin{aligned}
& \left|G_{1}\right| \leq C \varepsilon^{1-2 \beta}\left(\int_{0}^{t}\left\|\partial_{\rho} u_{1}\left(\frac{s}{\varepsilon^{\beta}}, x, \frac{x}{\varepsilon}\right)\right\|_{L^{2}\left(\mathbb{R}_{\varepsilon}^{d}\right)}^{2}\right)^{1 / 2}\left(\int_{0}^{t}\left\|\beta^{\varepsilon}(s)\right\|_{L^{2}\left(\mathbb{R}_{\varepsilon}^{d}\right)}^{2}\right)^{1 / 2} \\
& \leq C \varepsilon^{2-2 \beta}\left(\int_{0}^{t}\left\|\partial_{\rho} u_{1}\left(\frac{s}{\varepsilon^{\beta}}, x, \frac{x}{\varepsilon}\right)\right\|_{L^{2}\left(\mathbb{R}_{\varepsilon}^{d}\right)}^{2}\right)^{1 / 2}\left(\int_{0}^{t}\left\|\nabla \beta^{\varepsilon}(s)\right\|_{L^{2}\left(\mathbb{R}_{\varepsilon}^{d}\right)}^{2}\right)^{1 / 2} \\
& \leq C \varepsilon^{4-5 \beta} \int_{0}^{t}\left\|\partial_{\rho} u_{1}\left(\frac{s}{\varepsilon^{\beta}}, x, \frac{x}{\varepsilon}\right)\right\|_{L^{2}\left(\mathbb{R}_{\varepsilon}^{d}\right)}^{2}+\frac{\varepsilon^{\beta}}{8} \int_{0}^{t}\left\|\nabla \beta^{\varepsilon}(s)\right\|_{L^{2}\left(\mathbb{R}_{\varepsilon}^{d}\right)}^{2} \\
& \leq C \varepsilon^{4-4 \beta}+\frac{\varepsilon^{\beta}}{8} \int_{0}^{t}\left\|\nabla \beta^{\varepsilon}(s)\right\|_{L^{2}\left(\mathbb{R}_{\varepsilon}^{d}\right)}^{2} \\
& \left|G_{2}\right| \leq C \varepsilon^{\beta}\left(\int_{0}^{t}\left\|\triangle_{x} u_{0}\left(\frac{s}{\varepsilon^{2-\beta}}, x, \frac{x}{\varepsilon}\right)\right\|_{L^{2}\left(\mathbb{R}_{\varepsilon}^{d}\right)}^{2}\right)^{1 / 2}\left(\int_{0}^{t}\left\|\beta^{\varepsilon}(s)\right\|_{L^{2}\left(\mathbb{R}_{\varepsilon}^{d}\right)}^{2}\right) \\
& \leq C \varepsilon^{\beta+1}\left(\int_{0}^{t}\left\|\triangle_{x} u_{0}\left(\frac{s}{\varepsilon^{2-\beta}}, x, \frac{x}{\varepsilon}\right)\right\|_{L^{2}\left(\mathbb{R}_{\varepsilon}^{d}\right)}^{2}\right)^{1 / 2}\left(\int_{0}^{t}\left\|\nabla \beta^{\varepsilon}(s)\right\|_{L^{2}\left(\mathbb{R}_{\varepsilon}^{d}\right)}^{2}\right) \\
& \leq C \varepsilon^{2+\beta} \int_{0}^{t}\left\|\triangle_{x} u_{0}\left(\frac{s}{\varepsilon^{2-\beta}}, x, \frac{x}{\varepsilon}\right)\right\|_{L^{2}\left(\mathbb{R}_{\varepsilon}^{d}\right)}^{2}+\frac{\varepsilon^{\beta}}{8} \int_{0}^{t}\left\|\nabla \beta^{\varepsilon}(s)\right\|_{L^{2}\left(\mathbb{R}_{\varepsilon}^{d}\right)}^{2} \\
& \leq C \varepsilon^{4}+\frac{\varepsilon^{\beta}}{8} \int_{0}^{t}\left\|\nabla \beta^{\varepsilon}(s)\right\|_{L^{2}\left(\mathbb{R}_{\varepsilon}^{d}\right)}^{2} \\
& \left|G_{3}\right| \leq C \varepsilon^{\beta-1}\left(\int_{0}^{t}\left\|\operatorname{div}_{y} \nabla_{x} u_{0}\left(\frac{s}{\varepsilon^{2-\beta}}, x, \frac{x}{\varepsilon}\right)\right\|_{L^{2}\left(\mathbb{R}_{\varepsilon}^{d}\right)}^{2}\right)^{1 / 2}\left(\int_{0}^{t}\left\|\beta^{\varepsilon}(s)\right\|_{L^{2}\left(\mathbb{R}_{\varepsilon}^{d}\right)}^{2}\right)^{1 / 2} \\
& \leq C \varepsilon^{\beta}\left(\int_{0}^{t}\left\|\operatorname{div}_{y} \nabla_{x} u_{0}\left(\frac{s}{\varepsilon^{2-\beta}}, x, \frac{x}{\varepsilon}\right)\right\|_{L^{2}\left(\mathbb{R}_{\varepsilon}^{d}\right)}^{2}\right)^{1 / 2}\left(\int_{0}^{t}\left\|\nabla \beta^{\varepsilon}(s)\right\|_{L^{2}\left(\mathbb{R}_{\varepsilon}^{d}\right)}^{2}\right)^{1 / 2} \\
& \leq C \varepsilon^{\beta} \int_{0}^{t}\left\|\operatorname{div}_{y} \nabla_{x} u_{0}\left(\frac{s}{\varepsilon^{2-\beta}}, x, \frac{x}{\varepsilon}\right)\right\|_{L^{2}\left(\mathbb{R}_{\varepsilon}^{d}\right)}^{2}+\frac{\varepsilon^{\beta}}{8} \int_{0}^{t}\left\|\nabla \beta^{\varepsilon}(s)\right\|_{L^{2}\left(\mathbb{R}_{\varepsilon}^{d}\right)}^{2} \\
& \leq C \varepsilon^{2}+\frac{\varepsilon^{\beta}}{8} \int_{0}^{t}\left\|\nabla \beta^{\varepsilon}(s)\right\|_{L^{2}\left(\mathbb{R}_{\varepsilon}^{d}\right)}^{2} \\
& \left|G_{4}\right| \leq \varepsilon\left(\int_{0}^{t}\left\|\triangle_{x} u_{1}\left(\frac{s}{\varepsilon^{\beta}}, x, \frac{x}{\varepsilon}\right)\right\|_{L^{2}\left(\mathbb{R}_{\varepsilon}^{d}\right)}^{2}\right)^{1 / 2}\left(\int_{0}^{t}\left\|\beta^{\varepsilon}(s)\right\|_{L^{2}\left(\mathbb{R}_{\varepsilon}^{d}\right)}^{2}\right)^{1 / 2} \\
& \leq \varepsilon^{2}\left(\int_{0}^{t}\left\|\triangle_{x} u_{1}\left(\frac{s}{\varepsilon^{\beta}}, x, \frac{x}{\varepsilon}\right)\right\|_{L^{2}\left(\mathbb{R}_{\varepsilon}^{d}\right)}^{2}\right)^{1 / 2}\left(\int_{0}^{t}\left\|\nabla \beta^{\varepsilon}(s)\right\|_{L^{2}\left(\mathbb{R}_{\varepsilon}^{d}\right)}^{2}\right)^{1 / 2} \\
& \leq \varepsilon^{4-\beta} \int_{0}^{t}\left\|\triangle_{x} u_{1}\left(\frac{s}{\varepsilon^{\beta}}, x, \frac{x}{\varepsilon}\right)\right\|_{L^{2}\left(\mathbb{R}_{\varepsilon}^{d}\right)}^{2}+\frac{\varepsilon^{\beta}}{8} \int_{0}^{t}\left\|\nabla \beta^{\varepsilon}(s)\right\|_{L^{2}\left(\mathbb{R}_{\varepsilon}^{d}\right)}^{2} \\
& \leq C \varepsilon^{4}+\frac{\varepsilon^{\beta}}{8} \int_{0}^{t}\left\|\nabla \beta^{\varepsilon}(s)\right\|_{2}^{2} \text {, }
\end{aligned}
$$




$$
\begin{aligned}
&\left|G_{5}\right| \leq C\left(\int_{0}^{t}\left\|\operatorname{div}_{y} \nabla_{x} u_{1}\left(\frac{s}{\varepsilon^{\beta}}, x, \frac{x}{\varepsilon}\right)\right\|_{L^{2}\left(\mathbb{R}_{\varepsilon}^{d}\right)}^{2}\right)^{1 / 2}\left(\int_{0}^{t}\left\|\beta^{\varepsilon}(s)\right\|_{L^{2}\left(\mathbb{R}_{\varepsilon}^{d}\right)}^{2}\right)^{1 / 2} \\
& \leq C \varepsilon\left(\int_{0}^{t}\left\|\operatorname{div}_{y} \nabla_{x} u_{1}\left(\frac{s}{\varepsilon^{\beta}}, x, \frac{x}{\varepsilon}\right)\right\|_{L^{2}\left(\mathbb{R}_{\varepsilon}^{d}\right)}^{2}\right)^{1 / 2}\left(\int_{0}^{t}\left\|\nabla \beta^{\varepsilon}(s)\right\|_{L^{2}\left(\mathbb{R}_{\varepsilon}^{d}\right)}^{2}\right)^{1 / 2} \\
& \leq C \varepsilon^{2-\beta} \int_{0}^{t}\left\|\operatorname{div}_{y} \nabla_{x} u_{1}\left(\frac{s}{\varepsilon^{\beta}}, x, \frac{x}{\varepsilon}\right)\right\|_{L^{2}\left(\mathbb{R}_{\varepsilon}^{d}\right)}^{2}+\frac{\varepsilon^{\beta}}{8} \int_{0}^{t}\left\|\nabla \beta^{\varepsilon}(s)\right\|_{L^{2}\left(\mathbb{R}_{\varepsilon}^{d}\right)}^{2} \\
& \leq C \varepsilon^{2}+\frac{\varepsilon^{\beta}}{8} \int_{0}^{t}\left\|\nabla \beta^{\varepsilon}(s)\right\|_{L^{2}\left(\mathbb{R}_{\varepsilon}^{d}\right)}^{2}, \\
&\left|G_{6}\right| \leq C\left(\int_{0}^{t}\left\|\nabla_{x} p_{0}\left(\frac{s}{\varepsilon^{\beta}}, x, \frac{x}{\varepsilon}\right)\right\|_{L^{2}\left(\mathbb{R}_{\varepsilon}^{d}\right)}^{2}\right)^{1 / 2}\left(\int_{0}^{t}\left\|\beta^{\varepsilon}(s)\right\|_{L^{2}\left(\mathbb{R}_{\varepsilon}^{d}\right)}^{2}\right)^{1 / 2} \\
& \leq C \varepsilon\left(\int_{0}^{t}\left\|\nabla_{x} p_{0}\left(\frac{s}{\varepsilon^{\beta}}, x, \frac{x}{\varepsilon}\right)\right\|_{L^{2}\left(\mathbb{R}_{\varepsilon}^{d}\right)}^{2}\right)^{1 / 2}\left(\int_{0}^{t}\left\|\nabla \beta^{\varepsilon}(s)\right\|_{L^{2}\left(\mathbb{R}_{\varepsilon}^{d}\right)}^{2}\right)^{1 / 2} \\
& \leq C \varepsilon^{2-\beta} \int_{0}^{t}\left\|\nabla_{x} p_{0}\left(\frac{s}{\varepsilon^{\beta}}, x, \frac{x}{\varepsilon}\right)\right\|_{L^{2}\left(\mathbb{R}_{\varepsilon}^{d}\right)}^{2}+\frac{\varepsilon^{\beta}}{8} \int_{0}^{t}\left\|\nabla \beta^{\varepsilon}(s)\right\|_{L^{2}\left(\mathbb{R}_{\varepsilon}^{d}\right)}^{2} \\
& \leq C \varepsilon^{2}+\frac{\varepsilon^{\beta}}{8} \int_{0}^{t}\left\|\nabla \beta^{\varepsilon}(s)\right\|_{L^{2}\left(\mathbb{R}_{\varepsilon}^{d}\right)}^{2} .
\end{aligned}
$$

We remark that the final inequality for the term $G_{1}$ follows from the time estimates given in (4.17), while the final bounds in $G_{4}, G_{5}, G_{6}$ follow from (4.18) and the Poincaré inequality (4.23) on the domain $Y_{f}$.

In estimating the final term $G_{7}$, we have

$$
\begin{aligned}
\left|G_{7}\right| & =\varepsilon^{1-\beta}\left|\int_{0}^{t} p_{1}\left(\frac{s}{\varepsilon^{\beta}}, x, \frac{x}{\varepsilon}\right) \operatorname{div} \beta^{\varepsilon}\right| \\
& \leq C \varepsilon^{1-\beta}\left(\int_{0}^{t}\left\|p_{1}\left(\frac{s}{\varepsilon^{2-\beta}}, x, \frac{x}{\varepsilon}\right)\right\|_{L^{2}\left(\mathbb{R}_{\varepsilon}^{d}\right)}^{2}\right)^{1 / 2}\left(\int_{0}^{t}\left\|\nabla \beta^{\varepsilon}(s)\right\|_{L^{2}\left(\mathbb{R}_{\varepsilon}^{d}\right)}^{2}\right)^{1 / 2} \\
& \leq C \varepsilon^{2-3 \beta} \int_{0}^{t}\left\|p_{1}\left(\frac{s}{\varepsilon^{2-\beta}}, x, \frac{x}{\varepsilon}\right)\right\|_{L^{2}\left(\mathbb{R}_{\varepsilon}^{d}\right)}^{2}+\frac{\varepsilon^{\beta}}{8} \int_{0}^{t}\left\|\nabla \beta^{\varepsilon}(s)\right\|_{L^{2}\left(\mathbb{R}_{\varepsilon}^{d}\right)}^{2} \\
& \leq C \varepsilon^{4-4 \beta}+\frac{\varepsilon^{\beta}}{8} \int_{0}^{t}\left\|\nabla \beta^{\varepsilon}(s)\right\|_{L^{2}\left(\mathbb{R}_{\varepsilon}^{d}\right)}^{2} .
\end{aligned}
$$

We now explain the final inequality in (4.24). In order to do so, we need to obtain appropriate energy bounds for $u_{0}$. Multiplying the heat Equation (2.8) by $-\Delta_{y} u_{0}$ we obtain

$$
\left\|\nabla u_{0}(\tau, x, \cdot)\right\|_{L^{2}\left(Y_{f}\right)}^{2}+\frac{C}{2} \frac{d}{d \tau}\left\|\nabla u_{0}(\tau, x, \cdot)\right\|_{L^{2}\left(Y_{f}\right)}^{2} \leq 0,
$$

where we have made use of the fact

$$
\left\|u_{0}(\tau, x, \cdot)\right\|_{H_{0 \#}^{1}\left(Y_{f}\right)}=\left\|\triangle_{D}^{-1} \triangle u_{0}(\tau, x, \cdot)\right\|_{H_{0 \#}^{1}\left(Y_{f}\right)} \leq C\left\|\triangle u_{0}(\tau, x, \cdot)\right\|_{L^{2}\left(Y_{f}\right)} .
$$

We now apply Gronwall's lemma to (4.25) and deduce

$$
\left\|\nabla u_{0}(\tau, x, \cdot)\right\|_{L^{2}\left(Y_{f}\right)} \leq e^{-\tau / C}\left\|\nabla a_{0}(x, \cdot)\right\|_{L^{2}\left(Y_{f}\right)} .
$$


The inequality (4.24) now follows from the bound

$$
\left\|p_{1}\left(\frac{t}{\varepsilon^{2-\beta}}, x, \frac{x}{\varepsilon}\right)\right\|_{L^{2}\left(\mathbb{R}_{\varepsilon}^{d}\right)} \leq \int_{t / \varepsilon^{2-\beta}}^{\infty}\left\|\operatorname{div} u_{0}\left(\tau^{\prime}, x, \frac{x}{\varepsilon}\right)\right\|_{L^{2}\left(\mathbb{R}_{\varepsilon}^{d}\right)} \leq C_{1} e^{-C_{2} t / \varepsilon^{2-\beta}}
$$

We now combine all the estimates associated with the scalar and vector force terms, and notice that (4.22) holds with $\gamma=\min (2,4-4 \beta)$ and $C_{1}=7 / 8$. The proof of Theorem 3.3 is now complete.

\section{4. $(\beta=1)$ Proof of Theorem 3.5.}

Proof. We define the asymptotic expansions

$$
\begin{aligned}
\alpha^{\varepsilon} & =p^{\varepsilon}-p_{0}\left(\frac{t}{\varepsilon}, x, \frac{x}{\varepsilon}\right), \\
\beta^{\varepsilon} & =u^{\varepsilon}-u_{0}\left(\frac{t}{\varepsilon}, x, \frac{x}{\varepsilon}\right),
\end{aligned}
$$

where $\left(p_{0}, u_{0}\right)$ is the unique solution to the limit system (3.4). The expansions $\left(\alpha^{\varepsilon}, \beta^{\varepsilon}\right)$ satisfy (4.1), where the force terms are given by

$$
\begin{aligned}
& F^{\varepsilon}(t, x)=-\operatorname{div}_{x} u_{0}, \\
& G^{\varepsilon}(t, x)=\varepsilon \triangle_{x} u_{0}+2 \operatorname{div}_{y} \nabla_{x} u_{0}-\nabla_{x} p_{0},
\end{aligned}
$$

and similar to the cases $\beta<1$, it suffices to show (4.22).

In order to obtain the necessary bounds, we note that the solution $\left(p_{0}, u_{0}\right)$ satisfies the energy estimate

$$
\left\|p_{0}(t)\right\|_{L^{2}\left(Y_{f}\right)}^{2}+\left\|u_{0}(t)\right\|_{L^{2}\left(Y_{f}\right)}^{2}+2 \int_{0}^{t}\left\|\nabla_{y} u_{0}(s)\right\|_{L^{2}\left(Y_{f}\right)}^{2}=\left\|a_{0}\right\|_{L^{2}\left(Y_{f}\right)}^{2}+\left\|b_{0}(t)\right\|_{L^{2}\left(Y_{f}\right)}^{2} .
$$

As a result of (4.26), we have

$$
\begin{aligned}
\left|\int_{0}^{t} \int_{\mathbb{R}_{\varepsilon}^{d}} F^{\varepsilon} \alpha^{\varepsilon}\right| & \leq C \int_{0}^{t}\left\|\operatorname{div}_{x} u_{0}\left(\frac{s}{\varepsilon}, x, \frac{x}{\varepsilon}\right)\right\|_{L^{2}\left(\mathbb{R}_{\varepsilon}^{d}\right)}^{2}+\int_{0}^{t}\left\|\alpha^{\varepsilon}(t)\right\|_{L^{2}\left(\mathbb{R}_{\varepsilon}^{d}\right)}^{2} \\
& \leq C \varepsilon+\int_{0}^{t}\left\|\alpha^{\varepsilon}(t)\right\|_{L^{2}\left(\mathbb{R}_{\varepsilon}^{d}\right)}^{2} .
\end{aligned}
$$

We now estimate the term

$$
\int_{0}^{t} \int_{\mathbb{R}_{\varepsilon}^{d}} G^{\varepsilon} \cdot \beta^{\varepsilon}=G_{1}+G_{2}+G_{3}
$$


and obtain the following bounds:

$$
\begin{aligned}
\left|G_{1}\right| & \leq C \varepsilon\left(\int_{0}^{t}\left\|\triangle_{x} u_{0}\left(\frac{s}{\varepsilon}, x, \frac{x}{\varepsilon}\right)\right\|_{L^{2}\left(\mathbb{R}_{\varepsilon}^{d}\right)}^{2}\right)^{1 / 2}+\left(\int_{0}^{t}\left\|\beta^{\varepsilon}(s)\right\|_{L^{2}\left(\mathbb{R}_{\varepsilon}^{d}\right)}^{2}\right)^{1 / 2} \\
& \leq C \varepsilon^{2}\left(\int_{0}^{t}\left\|\triangle_{x} u_{0}\left(\frac{s}{\varepsilon}, x, \frac{x}{\varepsilon}\right)\right\|_{L^{2}\left(\mathbb{R}_{\varepsilon}^{d}\right)}^{2}\right)^{1 / 2}+\left(\int_{0}^{t}\left\|\nabla \beta^{\varepsilon}(s)\right\|_{L^{2}\left(\mathbb{R}_{\varepsilon}^{d}\right)}^{2}\right)^{1 / 2} \\
& \leq C \varepsilon^{3} \int_{0}^{t}\left\|\triangle_{x} u_{0}\left(\frac{s}{\varepsilon}, x, \frac{x}{\varepsilon}\right)\right\|_{L^{2}\left(\mathbb{R}_{\varepsilon}^{d}\right)}^{2}+\frac{\varepsilon}{4} \int_{0}^{t}\left\|\nabla \beta^{\varepsilon}(s)\right\|_{L^{2}\left(\mathbb{R}_{\varepsilon}^{d}\right)}^{2} \\
& \leq C \varepsilon^{4}+\frac{\varepsilon}{4} \int_{0}^{t}\left\|\nabla \beta^{\varepsilon}(s)\right\|_{L^{2}\left(\mathbb{R}_{\varepsilon}^{d}\right)}^{2}, \\
\left|G_{2}\right| & \leq C\left(\int_{0}^{t}\left\|\operatorname{div}_{y} \nabla_{x} u_{0}\left(\frac{s}{\varepsilon}, x, \frac{x}{\varepsilon}\right)\right\|_{L^{2}\left(\mathbb{R}_{\varepsilon}^{d}\right)}^{2}\right)^{1 / 2}\left(\int_{0}^{t}\left\|\beta^{\varepsilon}(s)\right\|_{L^{2}\left(\mathbb{R}_{\varepsilon}^{d}\right)}^{2}\right)^{1 / 2} \\
& \leq C \varepsilon\left(\int_{0}^{t}\left\|\operatorname{div}_{y} \nabla_{x} u_{0}\left(\frac{s}{\varepsilon}, x, \frac{x}{\varepsilon}\right)\right\|_{L^{2}\left(\mathbb{R}_{\varepsilon}^{d}\right)}^{2}\right)^{1 / 2}\left(\int_{0}^{t}\left\|\nabla \beta^{\varepsilon}(s)\right\|_{L^{2}\left(\mathbb{R}_{\varepsilon}^{d}\right)}^{2}\right)^{1 / 2} \\
& \leq C \varepsilon \int_{0}^{t}\left\|\operatorname{div}_{y} \nabla_{x} u_{0}\left(\frac{s}{\varepsilon}, x, \frac{x}{\varepsilon}\right)\right\|_{L^{2}\left(\mathbb{R}_{\varepsilon}^{d}\right)}^{2}+\frac{\varepsilon}{4} \int_{0}^{t}\left\|\beta^{\varepsilon}(s)\right\|_{L^{2}\left(\mathbb{R}_{\varepsilon}^{d}\right)}^{2} \\
& \leq C \varepsilon^{2}+\frac{\varepsilon}{4} \int_{0}^{t}\left\|\nabla \beta^{\varepsilon}(s)\right\|_{L^{2}\left(\mathbb{R}_{\varepsilon}^{d}\right)}^{2}, \\
\left|G_{3}\right| & \leq C\left(\int_{0}^{t}\left\|\nabla_{x} p_{0}\left(\frac{s}{\varepsilon}, x, \frac{x}{\varepsilon}\right)\right\|_{L^{2}\left(\mathbb{R}_{\varepsilon}^{d}\right)}^{2}\right)^{1 / 2}\left(\int_{0}^{t}\left\|\beta^{\varepsilon}(s)\right\|_{L^{2}\left(\mathbb{R}_{\varepsilon}^{d}\right)}^{2}\right)^{1 / 2} \\
& \leq C \varepsilon\left(\int_{0}^{t}\left\|\nabla_{x} p_{0}\left(\frac{s}{\varepsilon}, x, \frac{x}{\varepsilon}\right)\right\|_{L^{2}\left(\mathbb{R}_{\varepsilon}^{d}\right)}^{2}\right)^{1 / 2}\left(\int_{0}^{t}\left\|\nabla \beta^{\varepsilon}(s)\right\|_{L^{2}\left(\mathbb{R}_{\varepsilon}^{d}\right)}^{2}\right)^{1 / 2} \\
& \leq C \varepsilon \int_{0}^{t}\left\|\nabla_{x} p_{0}\left(\frac{s}{\varepsilon}, x, \frac{x}{\varepsilon}\right)\right\|_{L^{2}\left(\mathbb{R}_{\varepsilon}^{d}\right)}^{2}+\frac{\varepsilon}{4} \int_{0}^{t}\left\|\nabla \beta^{\varepsilon}(s)\right\|_{L^{2}\left(\mathbb{R}_{\varepsilon}^{d}\right)}^{2} \\
& \leq C t \varepsilon \int_{0}^{t}\left\|\nabla \beta^{\varepsilon}(s)\right\|_{L^{2}\left(\mathbb{R}_{\varepsilon}^{d}\right)}^{2} \cdot .
\end{aligned}
$$

Combing the estimates associated to $F^{\varepsilon}$ and $G^{\varepsilon}$, we note that (4.22) holds with $\gamma=1$ and $C_{1}=3 / 4$. The proof of Theorem 3.5 is now complete.

\section{5. $(0<\beta<1)$ Proof of Corollary 3.8.}

Proof. In this subsection we explain some of the changes that must be made to the proof given in Subsection 4.3. Instead of (4.20), we define the following error terms:

$$
\begin{aligned}
& \alpha_{\delta}^{\varepsilon}=p^{\varepsilon}-p_{0} \star \omega_{\delta}\left(\frac{t}{\varepsilon^{\beta}}, x, \frac{x}{\varepsilon}\right)-\varepsilon^{(1-\beta)} p_{1} \star \omega_{\delta}\left(\frac{t}{\varepsilon^{2-\beta}}, x, \frac{x}{\varepsilon}\right), \\
& \beta_{\delta}^{\varepsilon}=u^{\varepsilon}-u_{0} \star \omega_{\delta}\left(\frac{t}{\varepsilon^{2-\beta}}, x, \frac{x}{\varepsilon}\right)-\varepsilon^{(1-\beta)} u_{1} \star \omega_{\delta}\left(\frac{t}{\varepsilon^{\beta}}, x, \frac{x}{\varepsilon}\right) .
\end{aligned}
$$

The error term $\left(\alpha_{\delta}^{\varepsilon}, \beta_{\delta}^{\varepsilon}\right)$ defined in (4.27) satisfies the system given in (4.1), with force terms $F_{\delta}^{\varepsilon}(t, x)=F^{\varepsilon} \star \omega_{\delta}(t, x)$ and $G_{\delta}^{\varepsilon}(t, x)=G^{\varepsilon} \star \omega_{\delta}(t, x)$, where $F^{\varepsilon}$ and $G^{\varepsilon}$ are defined in (4.21). Arguing as in (4.2) and (4.22) and using that $\left\|\nabla_{x}^{k}\left(u \star \omega_{\delta}\right)\right\|_{L^{2}\left(\mathbb{R}^{d} \times Y_{f}\right)} \leq$ 
$C \delta^{-k}\|u\|_{L^{2}\left(\mathbb{R}^{d} \times Y_{f}\right)}$ for any function $u(x, y)$, we deduce that

$$
\begin{gathered}
\left\|\alpha_{\delta}^{\varepsilon}(t)\right\|_{L^{2}\left(\mathbb{R}_{\varepsilon}^{d}\right)}^{2}+\left\|\beta_{\delta}^{\varepsilon}(t)\right\|_{L^{2}\left(\mathbb{R}_{\varepsilon}^{d}\right)}^{2}+\frac{\varepsilon^{\beta}}{4} \int_{0}^{t}\left\|\nabla \beta_{\delta}^{\varepsilon}(s)\right\|_{L^{2}\left(\mathbb{R}_{\varepsilon}^{d}\right)}^{2} \\
\leq\left\|\alpha_{\delta}^{\varepsilon}(0)\right\|_{L^{2}\left(\mathbb{R}_{\varepsilon}^{d}\right)}^{2}+\left\|\beta_{\delta}^{\varepsilon}(0)\right\|_{L^{2}\left(\mathbb{R}_{\varepsilon}^{d}\right)}^{2}+\int_{0}^{t}\left\|\alpha^{\varepsilon}(s)\right\|_{L^{2}\left(\mathbb{R}_{\varepsilon}^{d}\right)}^{2}+\frac{C \varepsilon^{\gamma}}{\delta^{2}}
\end{gathered}
$$

where $\gamma=\min (2,4-4 \beta)$. Hence, by Gronwall Lemma, we deduce that

$$
\begin{gathered}
\left\|\alpha_{\delta}^{\varepsilon}(t)\right\|_{L^{2}\left(\mathbb{R}_{\varepsilon}^{d}\right)}^{2}+\left\|\beta_{\delta}^{\varepsilon}(t)\right\|_{L^{2}\left(\mathbb{R}_{\varepsilon}^{d}\right)}^{2}+\frac{\varepsilon^{\beta}}{4} \int_{0}^{t}\left\|\nabla \beta_{\delta}^{\varepsilon}(s)\right\|_{L^{2}\left(\mathbb{R}_{\varepsilon}^{d}\right)}^{2} \\
\leq e^{C t}\left[\left\|\alpha_{\delta}^{\varepsilon}(0)\right\|_{L^{2}\left(\mathbb{R}_{\varepsilon}^{d}\right)}^{2}+\left\|\beta_{\delta}^{\varepsilon}(0)\right\|_{L^{2}\left(\mathbb{R}_{\varepsilon}^{d}\right)}^{2}+\frac{C \varepsilon^{\gamma}}{\delta^{2}}\right] .
\end{gathered}
$$

For each $\alpha>0$, there exists $\delta_{0}$ such that for $0<\delta<\delta_{0}$, we have

$$
\left\|a_{0}(x, y)-a_{0} \star \omega_{\delta}(x, y)\right\|_{L^{2}\left(\mathbb{R}^{d} \times Y_{f}\right)}+\left\|b_{0}(x, y)-b_{0} \star \omega_{\delta}(x, y)\right\|_{L^{2}\left(\mathbb{R}^{d} \times Y_{f}\right)}<\alpha .
$$

We fix such a $\delta$. Since $\left(a^{\varepsilon}, b^{\varepsilon}\right)$ two-scale converges strongly to $\left(a_{0}, b_{0}\right)$, we deduce that

$$
\begin{aligned}
& \left\|a^{\varepsilon}(x)-a_{0} \star \omega_{\delta}\left(x, \frac{x}{\varepsilon}\right)\right\|_{L^{2}\left(\mathbb{R}_{\varepsilon}^{d}\right)} \stackrel{\varepsilon \rightarrow 0}{\rightarrow}\left\|a_{0}(x, y)-a_{0} \star \omega_{\delta}(x, y)\right\|_{L^{2}\left(\mathbb{R}^{d} \times Y_{f}\right)}, \\
& \left\|b^{\varepsilon}(x)-b_{0} \star \omega_{\delta}\left(x, \frac{x}{\varepsilon}\right)\right\|_{L^{2}\left(\mathbb{R}_{\varepsilon}^{d}\right)} \stackrel{\varepsilon \rightarrow 0}{\rightarrow}\left\|b_{0}(x, y)-b_{0} \star \omega_{\delta}(x, y)\right\|_{L^{2}\left(\mathbb{R}^{d} \times Y_{f}\right)}
\end{aligned}
$$

when $\varepsilon$ goes to 0 . Hence, there exists an $\varepsilon_{0}$ such that for $0<\varepsilon<\varepsilon_{0}$, we have

$$
\left\|a^{\varepsilon}(x)-a_{0} \star \omega_{\delta}\left(x, \frac{x}{\varepsilon}\right)\right\|_{L^{2}\left(\mathbb{R}_{\varepsilon}^{d}\right)}+\left\|b^{\varepsilon}(x)-b_{0} \star \omega_{\delta}\left(x, \frac{x}{\varepsilon}\right)\right\|_{L^{2}\left(\mathbb{R}_{\varepsilon}^{d}\right)}<2 \alpha .
$$

Also, there exists an $\varepsilon_{1}$ such that for $0<\varepsilon<\varepsilon_{1}$, we have $\frac{\varepsilon^{\gamma}}{\delta^{2}}<\alpha$ (recall that $\delta$ has been fixed). Hence, for $0<\varepsilon<\min \left(\varepsilon_{0}, \varepsilon_{1}\right)$, we have

$$
\sup _{0 \leq t \leq T}\left[\left\|\alpha_{\delta}^{\varepsilon}(t)\right\|_{L^{2}\left(\mathbb{R}_{\varepsilon}^{d}\right)}^{2}+\left\|\beta_{\delta}^{\varepsilon}(t)\right\|_{L^{2}\left(\mathbb{R}_{\varepsilon}^{d}\right)}^{2}\right]+\frac{\varepsilon^{\beta}}{4} \int_{0}^{T}\left\|\nabla \beta_{\delta}^{\varepsilon}(s)\right\|_{L^{2}\left(\mathbb{R}_{\varepsilon}^{d}\right)}^{2}<3 e^{C T} \alpha,
$$

and hence Corollary 3.8 is proved.

\section{Concluding remarks}

We remark on our forthcoming papers [7,6], i.e., the case $\beta>1$. To see the difficulty in dealing with time-space boundary layers, we present a formal argument in the case $\beta=2$. Assuming the following two-scale asymptotic expansion for the pressure and velocity

$$
\begin{aligned}
& p^{\varepsilon}=\sum_{j=0}^{\infty} \varepsilon^{j} p_{j}\left(t, x, \frac{x}{\varepsilon}\right), \\
& u^{\varepsilon}=\sum_{j=0}^{\infty} \varepsilon^{j} u_{j}\left(t, x, \frac{x}{\varepsilon}\right),
\end{aligned}
$$


we conclude the following system for terms $\left(p_{0}, p_{1}, u_{0}\right)$ :

$$
\left\{\begin{aligned}
\left|Y_{f}\right| \partial_{t} p_{0}+\operatorname{div}_{x} \int_{Y_{f}} u_{0} & =0 & & \text { in } \mathbb{R}^{d}, \\
\partial_{t} u_{0}-\triangle_{y} u+\nabla_{x} p_{0}+\nabla_{y} p_{1} & =0 & & \\
\operatorname{div}_{y} u_{0} & =0, & & \\
\nabla_{y} p_{0} & =0, & & \mathbb{R}^{d} \times Y_{f}, \\
u_{0} & =0 & & \\
p_{0}(t=0) & =b(x), & & \\
u_{0}(t=0) & =P_{y} a_{0}(x, y), & & \\
y \rightarrow\left(u_{0}, p_{1}\right) & \text { is } & Y \text {-periodic, }, &
\end{aligned}\right.
$$

where $P_{y}$ is the usual Helmholtz operator on the domain $Y_{f}$. Notice that two-scale convergence only captures the micro-incompressible part of the flow. This is due to the formation of a thin boundary layer in time of size $\varepsilon^{1 / 2}$ which decouples the flow into its micro-incompressible and micro-acoustic parts. The micro-acoustic part of the flow, which we define as $\left(q^{\varepsilon}, v^{\varepsilon}\right)$, carries fast time oscillations, and the partition of the flow near initial time can be seen in Figure 5.1 (compare with Figure 1.4). Notice

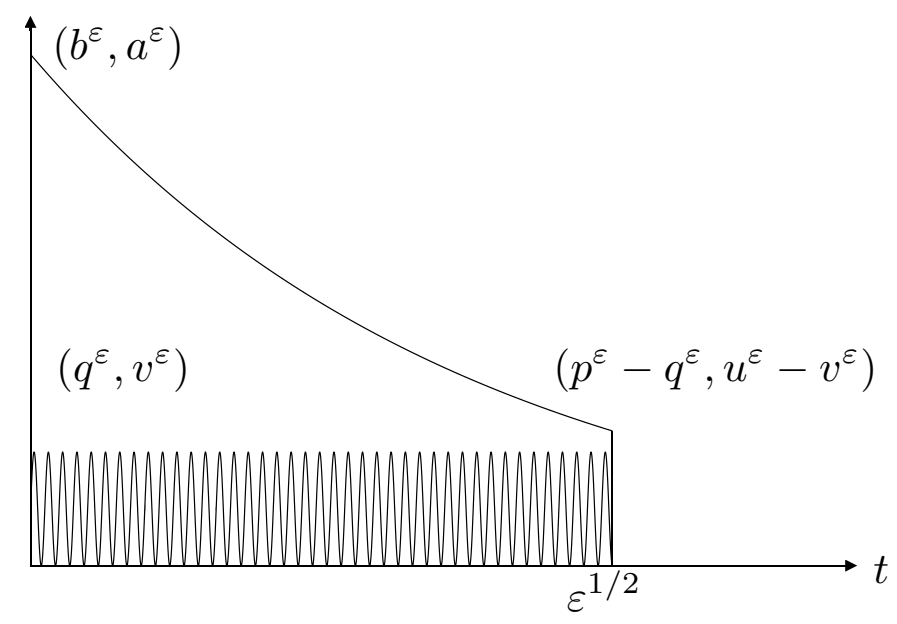

FIG. 5.1. Boundary layer in time for $\beta=2$.

that the fast time oscillations associated to micro-acoustic flow are quickly damped in the case $\beta=2$.

How to split the flow into its incompressible and acoustic parts on the microscopic scale is performed in our second paper [7]. In addition to two-scale convergence, we make use of the Bloch decomposition of the wave operator. By this we mean the spectral decomposition of the wave operator

$$
A_{0}=\left(\begin{array}{cc}
0 & \operatorname{div} \\
\nabla & 0
\end{array}\right)
$$

on the elementary fluid cell $Y_{f}$ equipped with $\theta$-quasi periodic boundary conditions. 
We conclude our study of the total energy (1.2) in our final paper [6] by describing the energy carried by the acoustic part of the flow. More precisely, we provide appropriate asymptotic expansions for $\left(q^{\varepsilon}, v^{\varepsilon}\right)$ via the Bloch decomposition of the viscous wave operator

$$
A^{\varepsilon^{\beta}}=-\left(\begin{array}{cc}
0 & \text { div } \\
\nabla & 0
\end{array}\right)+\varepsilon^{\beta}\left(\begin{array}{ll}
0 & 0 \\
0 & \triangle
\end{array}\right) .
$$

Closed equations describing micro-acoustic flow near initial time are obtained using the Wigner measure.

Appendix A. Dimensional analysis. We consider a compressible fluid governed by the isentropic compressible Navier-Stokes equation. We study its flow in a porous medium $\mathbb{R}_{l}^{d}$, where $l$ is the size of each cell.

$$
\left\{\begin{array}{l}
\partial_{t} \rho+\operatorname{div}(\rho u)=0, \quad \rho \geq 0, \\
\partial_{t}(\rho u)+\operatorname{div}(\rho u \otimes u)-\mu \Delta u-\xi \nabla \operatorname{div} u+\nabla p=0,
\end{array}\right.
$$

with the Dirichlet boundary condition $u=0$ on the boundary $\partial \mathbb{R}_{l}^{d}$. Here, $\rho$ and $u$ are respectively the density and the velocity of the fluid and the pressure $p$ is given by a baroscopic law $p=a \rho^{\gamma}, a$ is a constant related to the entropy and $\mu$ is the viscosity. We would like to look at this model on a macroscopic scale $L$ which is much larger than the length $l$ of each cell. Hence, we introduce the nondimensional parameter $\varepsilon=\frac{l}{L}$. We also introduce a time scale $T$ and a velocity scale $U$. Since we are looking at the propagation of acoustic waves with a fixed background, we will assume that $\rho=\bar{\rho}(1+\alpha \tilde{\rho})$ where $\alpha$ is a small parameter, namely $\rho$ is a perturbation of order $\alpha$ of a fixed background $\bar{\rho}$. Hence, the sound speed $c$ is given by $c^{2}=a \gamma \bar{\rho}^{\gamma-1}$. In this problem we have two typical speeds, namely the sound speed $c$ and the velocity scale $U$. Since we want to neglect the nonlinear effect coming from the transport term, we take $U \ll c$ and define $\kappa=\frac{U}{c}$. We also adjust the time scale $T$ in such a way that $c=\frac{L}{T}$. We introduce the adimensional quantities $\tilde{t}, \tilde{x}, \ldots$ given by

$$
t=T \tilde{t}, \quad x=L \tilde{x}, \quad u=U \tilde{u} .
$$

Hence, (A.1) becomes

$$
\left\{\begin{array}{l}
\partial_{\tilde{t}} \tilde{\rho}+\frac{T U}{\alpha L} \tilde{\operatorname{div}}(\tilde{u})+\frac{U T}{L} \tilde{\operatorname{div}}(\tilde{\rho} \tilde{u})=0, \quad \rho \geq 0, \\
\partial_{\hat{t}} \tilde{u}+\frac{U T}{L} \tilde{u} \tilde{\nabla} \tilde{u}-\frac{\mu T}{\rho L^{2}} \tilde{\Delta} u-\frac{\xi T}{\rho L^{2}} \tilde{\nabla} \tilde{\operatorname{div}} u+\frac{\alpha T c^{2}}{U L} \tilde{\nabla} \tilde{\rho}+\frac{T}{L U} \frac{a \gamma}{\gamma-1} \nabla \pi=0,
\end{array}\right.
$$

where $\pi=\bar{\rho}^{\gamma-1}\left[(1+\alpha \tilde{\rho})^{\gamma-1}-1-\alpha(\gamma-1) \tilde{\rho}\right]$.

Recall that $T$ and $L$ are chosen in such a way that $c=\frac{L}{T} \gg U$. Also the fluctuation in the density and the velocity scale $U$ should be related by the fact that $\alpha=\kappa$, namely $\alpha=\frac{U}{c}$. For simplicity, we will also take $\xi=0$. Hence, (A.3) becomes

$$
\left\{\begin{array}{l}
\partial_{\tilde{t}} \tilde{\rho}+\tilde{\operatorname{div}}(\tilde{u})+\alpha \tilde{\operatorname{div}}(\tilde{\rho} \tilde{u})=0, \quad \rho \geq 0, \\
\partial_{\tilde{t}} \tilde{u}+\alpha \tilde{u} \tilde{\nabla} \tilde{u}-\frac{\mu T}{\bar{\rho} L^{2}} \tilde{\Delta} u+\tilde{\nabla} \tilde{\rho}+\frac{a \gamma}{\alpha(\gamma-1)} \nabla \pi+\left(\frac{\mu T}{\bar{\rho} L^{2}}-\frac{\mu T}{\rho L^{2}}\right) \tilde{\Delta} u=0 .
\end{array}\right.
$$

Here, we are interested in the understanding of the linear propagation and this is why we took $\alpha \ll 1$, which allows us to neglect the nonlinear terms; notice that they all 
have a factor $\alpha$ in front. Neglecting the nonlinear terms, (A.4) becomes

$$
\left\{\begin{array}{l}
\partial_{\tilde{t}} \tilde{\rho}+\tilde{\operatorname{div}}(\tilde{u})=0, \quad \rho \geq 0, \\
\partial_{\tilde{t}} \tilde{u}-\frac{\mu T}{\bar{\rho} L^{2}} \tilde{\Delta} u+\tilde{\nabla} \tilde{\rho}=0 .
\end{array}\right.
$$

Denoting $\left(u^{\varepsilon}, p^{\varepsilon}\right)=(\tilde{u}, \tilde{\rho})$, dropping the tilde from $\tilde{t}, \tilde{x}$ and defining $\varepsilon^{\beta}=\frac{\mu T}{\bar{\rho} L^{2}}$, we get the system (1.1).

Recall that the Reynolds number is defined by $R e=\frac{\bar{\rho} U L}{\mu}$. It measure the relative importance of the transport effect to the viscous effects. One can define a modified Reynolds number $R e_{c}=\frac{\bar{\rho} c L}{\mu}=\varepsilon^{-\beta}$ that measures the relative importance of the acoustic effect to the viscous effects.

\section{REFERENCES}

[1] G. Allaire, Homogenization of the Stokes flow in a connected porous medium, Asymp. Anal., 2(3), 203-222, 1989.

[2] G. Allaire, Homogenization and two-scale convergence, SIAM J. Math. Anal., 23(6), 1482-1518, 1992.

[3] A. Bensoussan, J.-L. Lions, and G. Papanicolaou, Asymptotic Analysis for Periodic Structures, North-Holland Publishing Co., Amsterdam, 1978.

[4] M.E. Bogovskiú, Solutions of some problems of vector analysis, associated with the operators div and grad, in Theory of Cubature Formulas and the Application of Functional Analysis to Problems of Mathematical Physics, Akad. Nauk SSSR Sibirsk. Otdel. Inst. Mat., Novosibirsk, 5-40, 149, 1980.

[5] J. Diaz-Alban, The High Frequency and Inviscid Limit of Acoustic Waves in a Porous Medium, Ph.D. Thesis, Courant Institute of Mathematical Sciences, New York University, 2008.

[6] J. Diaz-Alban and N. Masmoudi, The asymptotic analysis of acoustic waves in a porous medium: An application of the Wigner Measure, in preparation.

[7] J. Diaz-Alban and N. Masmoudi, The asymptotic analysis of acoustic wavs in a porous medium: Time-space boundary layers and two-scale convergence, in preparation.

[8] J.I. Díaz, Two problems in homogenization of porous media, in Proceedings of the Second International Seminar on Geometry, Continua and Microstructure (Getafe, 1998), 14, 141-155, 1999.

[9] E. Feireisl, A. Novotný, and T. Takahashi, Homogenization and singular limits for the complete Navier-Stokes-Fourier system, J. Math. Pures Appl., (9), 94(1), 33-57, 2010.

[10] G.P. Galdi, An Introduction to the Mathematical Theory of the Navier-Stokes Equations: Linearized Steady Problems, Springer-Verlag, New York, Vol. I, 1994.

[11] J.L. Lions, Some Methods in the Mathematical Analysis of Systems and Their Control, Kexue Chubanshe (Science Press), Beijing, 1981.

[12] P.L. Lions and N. Masmoudi, Homogenization of the Euler system in a $2 D$ porous medium, J. Math. Pures Appl., (9), 84(1), 1-20, 2005.

[13] N. Masmoudi, Homogenization of the compressible Navier-Stokes equations in a porous medium (A tribute to J. L. Lions.), ESAIM Control Optim. Calc. Var., 8, 885-906 (electronic), 2002.

[14] A. Mikelić, Homogenization of nonstationary Navier-Stokes equations in a domain with a grained boundary, Ann. Mat. Pura Appl., (4), 158, 167-179, 1991.

[15] E. Marušić-Paloka and M. Starčević, Asymptotic analysis of an isothermal gas flow through a long or thin pipe, Math. Models Methods Appl. Sci., 19(4), 631-649, 2009.

[16] G. Nguetseng, A general convergence result for a functional related to the theory of homogenization, SIAM J. Math. Anal., 20(3), 608-623, 1989.

[17] G. Nguetseng, Asymptotic analysis for a stiff variational problem arising in mechanics, SIAM J. Math. Anal., 21(6), 1394-1414, 1990.

[18] E. Sánchez-Palencia, Nonhomogeneous Media and Vibration Theory, Springer-Verlag, Berlin, 1980.

[19] L. Tartar, Incompressible fluid flow in a porous medium: Convergence of the homogenization process, in Nonhomogeneous Media and Vibration Theory (E. Sánchez-Palencia), 368-377, 1980.

[20] H. Zhao and Z.-A. Yao, Homogenization of a non-stationary Stokes flow in porous medium including a layer, J. Math. Anal. Appl., 342(1), 108-124, 2008. 